\title{
Performance improvement of a vibration driven system for marine vessels
}

\author{
Roberto Muscia \\ Department of Engineering and Architecture, University of Trieste, Trieste, ITALY
}

\begin{abstract}
In this paper some developments concerning the possibility of generating a rectilinear motion of bodies partially or totally submerged subject to vibration, without the use of propellers, are presented. The motion is obtained by a device equipped with counter rotating masses installed in the vessel which vibrates along the longitudinal direction. The hull has a suitably shaped stern. The study considers an analysis for evaluating the energy that the propulsion system consumes in relation to its performances. A further objective was to maximize the speed of the system while keeping certain parameters unchanged relating to the equations of motion of the device and suitably allocating the counter rotating masses. This result is obtained by using elliptical gears to transmit the motion from the driving motor to a double pair of counter rotating masses. Such a solution allows one to reach the variability of the angular velocity of the counter rotating masses during each revolution in accordance with certain laws that maximize the thrust applied to the vessel preferentially along a direction in respect of the opposite one, all being equal. Finally, a formulation to compute the propulsive efficiency of the device study and the results of the numerical simulations carried out are illustrated.
\end{abstract}

KEY WORDS: Dynamics; Vibrations; Motion equations; Centrifugal force; Propulsion system; CFD computation

\section{Introduction}

The working principle of a propulsion system based on vibrating devices subject to various kinds of friction and or drag forces that simultaneously hinder and cause the motion preferentially along a direction with respect to the opposite one has been studied by many authors [1-12]. These researchers have confirmed the real possibility to obtain a displacement along a certain direction. Also from the experimental point of view this possibility has been substantiated $[8,9]$. In relation to a possible practical application of the above-mentioned propulsion principle we observe that the state of the art in the field of marine propulsion essentially concerns the improvement and optimization of the hull-propeller system. With reference to this topic in-depth fluid dynamics simulation studies [13-21] have been developed over the years. Such studies concern the interaction between the shape of the stern and that of one or more rotating propellers with the general aim of improving the efficiency of the propulsion system. The kind of problem, even if simplifying assumptions, like the constant angular velocity of the propeller, are considered, it is highly complex and to get results that reflect, at least with a certain reliability, what could happen in reality, computers with very high performances must be utilised. Moreover, the cost of manufacturing and maintenance of the propellers of large dimensions is very high. Then, the attempt to evaluate if new marine propulsion systems are possible and convenient could be beneficial. From this point of view in [10] an analytical numerical study of a vibrating propulsion device that could be adopted to move bodies partially or totally submerged is illustrated. This device does not consider the utilisation of propellers, it uses centrifugal force that arises, for example, from the rotation of a pair of counter rotating masses. In particular, the resultant of the centrifugal forces acting on these masses causes the motion of the vessel. Such resultant acts along a fixed direction, the longitudinal direction of the hull, and oscillates from a minimum value to a maximum value. Since during each rotation of the counter rotating masses the resultant changes direction and the whole device is integral with the hull, the hull itself periodically receives a forward and a backward thrust. As the stern of the hull is suitably shaped, the hydrodynamic drag force during the backward motion is significantly higher 


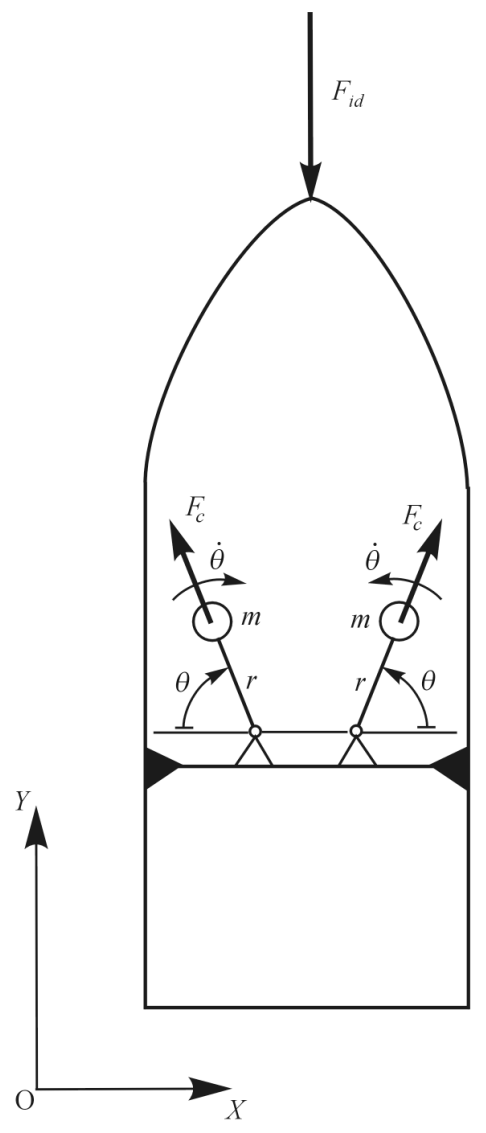

(a)

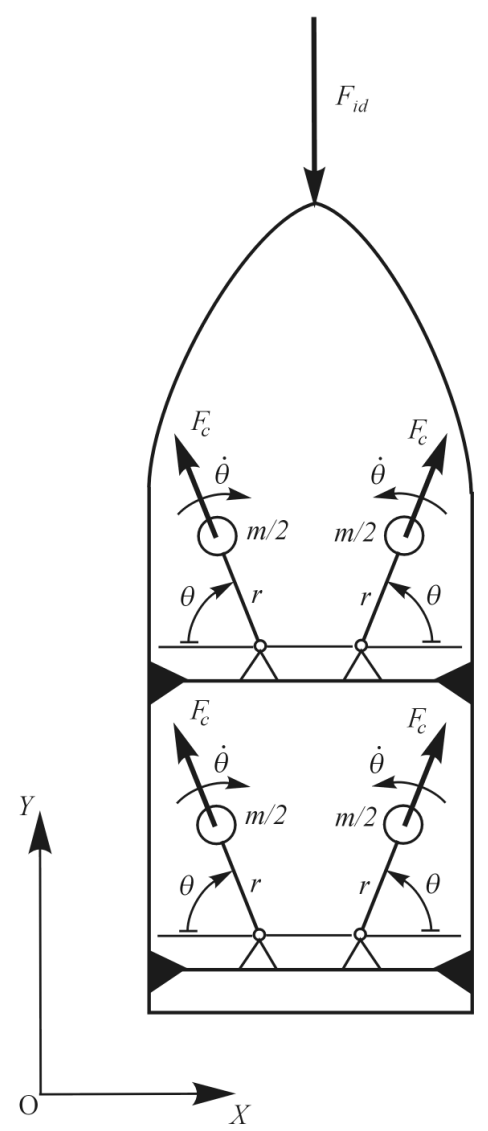

(b)

Fig. 1 Kinematic scheme of the propulsion system equipped with (a) one [10] and (b) two pairs of counter rotating masses.

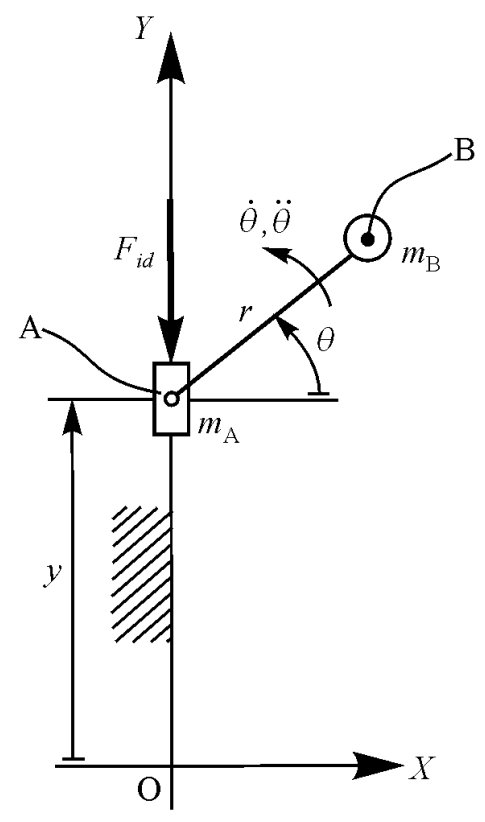

(a)

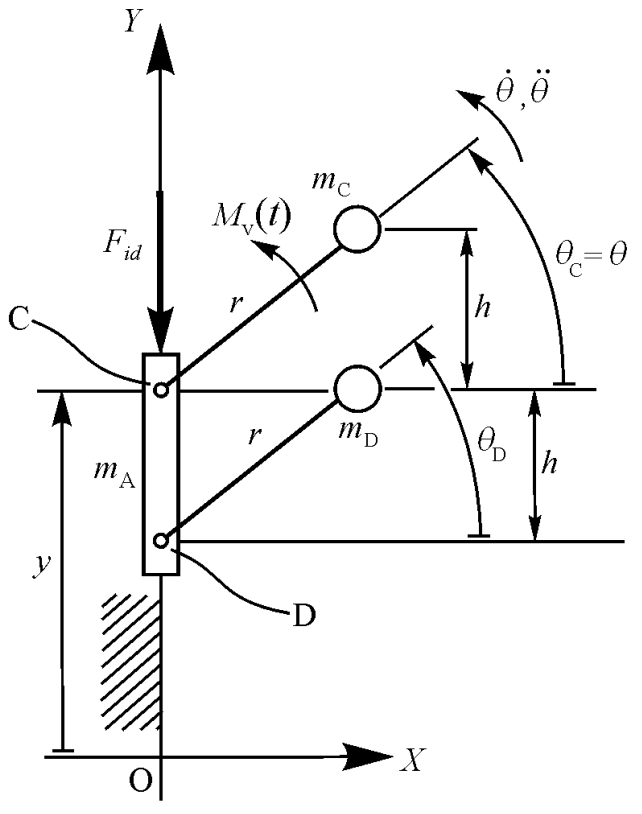

(b)

Fig. 2 Simplified kinematic scheme based on sliding fit of the propulsion system equipped with (a) one [10] and (b) two rotating masses. 
than that which is produced during the phase of forward moving. Consequently, a forward displacement resultant of the whole hull-device system corresponding to each complete rotation of the counter rotating masses is generated. The equations of motion of the system just described have been obtained in [10] and it has been shown that, by integrating such equations, actually the system behaves as illustrated. These results will be utilized to develope the improved propulsion device illustrated in the following paragraphs. This improved device is based on two important modifications of the orginal mechanism studied in [10]: i) the simple pair of counter rotating masses is replaced by two pairs of phased counter rotating masses and ii) the angular velocity of these two pairs of masses is not constant, but changes according to a periodic law that repeats itself for each rotation. Finally, the energy and efficiency issues of the propulsion system, which were not studied in [10], will be dealt with.

\section{Mechanical model}

\subsection{Another pair of counter rotating masses}

In relation to a possible manufacturing of a prototype of a vessel characterized by a length slightly greater than $1700 \mathrm{~mm}$, the results derived from numerical simulations of the system with one pair of counter rotating masses [10] (see Fig. 1(a)) showed high values of centrifugal force. From an engineering point of view, these values determine significant stress on the various mechanical components whereby the propulsion device has to be realized. This fact implies certainly dimensions and weight of the device itself that are not suitable for the need to limit the size of the whole structure. So, already in the preliminary study phase of the propulsion system, it is definitely convenient to consider a distribution of these masses suitably phased so as to reduce the strength of each centrifugal force that stresses the device. The simplest and feasible solution is to consider four counter rotating masses (see Fig. 1(b)) [5], each equal to $1 / 4$ of the total value $2 m$ of the rotating mass originally divided into only two masses $m$ (see Fig. 1(a)). In this way the equivalent system shown in Fig. 2(a) is replaced by that shown in Fig. 2(b). In Fig. 2(b) the masses $m_{\mathrm{C}}$ and $m_{\mathrm{D}}$ are two identical masses that replace the only mass $m_{\mathrm{B}}$ indicated in Fig. 2(a). If the original pair of counter rotating masses (see Fig. 1(a)) is replaced by two pairs of counter rotating masses each of them equal to $1 / 2$ of the each mass $m$ indicated in Fig. 1(a), it is apparent that, being equal radii of rotation and angular velocity of all the masses considered, the resultant of all the centrifugal forces along the $Y$ axis remains unchanged. So, if $m_{\mathrm{B}}=2 \mathrm{~m}$ is the mass of the kinematically equivalent system (Fig. 2(a)) to that one represented in Fig. 1(a), to obtain the same component of the centrifugal force along $Y$, the two masses $m_{\mathrm{C}}$ and $m_{\mathrm{D}}$, equal to each other, must have a value equal to $m_{\mathrm{B}} / 2$, that is $m_{\mathrm{C}}=m_{\mathrm{D}}=m_{\mathrm{B}} / 2$.

\subsection{Equations of motion of the system}

\subsubsection{Displacement analysis}

The equations of motion of the system shown in Figs. 1(b) and 2(b) are obtained by using the same procedure described in [10,5], that is by the Lagrange's equations. Denoting by $\theta_{\mathrm{C}}$ and $\theta_{\mathrm{D}}$ the angular coordinates of the respective masses $m_{\mathrm{C}}$ and $m_{\mathrm{D}}$, the abscissas $x_{\mathrm{C}}, x_{\mathrm{D}}$ and ordinates $y_{\mathrm{C}}, y_{\mathrm{D}}$ that identify the position of the same masses $m_{\mathrm{C}}$ and $m_{\mathrm{D}}$ are

$$
\begin{aligned}
& x_{\mathrm{C}}=r \cos \theta_{\mathrm{C}} \\
& y_{\mathrm{C}}=y+r \sin \theta_{\mathrm{C}}, \\
& x_{\mathrm{D}}=r \cos \theta_{\mathrm{D}}, \\
& y_{\mathrm{D}}=y-h+r \sin \theta_{\mathrm{D}},
\end{aligned}
$$


2.2.2 Velocity analysis and kinetic energy

Deriving Eqs. (1)-(3) with respect to time, the corresponding velocity of the masses $m_{\mathrm{C}}$ and $m_{\mathrm{D}}$ along the axes $X$ and $Y$ are obtained:

$$
\begin{aligned}
& \dot{x}_{\mathrm{C}}=-r \dot{\theta}_{\mathrm{C}} \sin \theta_{\mathrm{C}}, \\
& \dot{y}_{\mathrm{C}}=\dot{y}+r \dot{\theta}_{\mathrm{C}} \cos \theta_{\mathrm{C}}, \\
& \dot{x}_{\mathrm{D}}=-r \dot{\theta}_{\mathrm{D}} \sin \theta_{\mathrm{D}}, \\
& \dot{y}_{\mathrm{D}}=\dot{y}+r \dot{\theta}_{\mathrm{D}} \cos \theta_{\mathrm{D}}
\end{aligned}
$$

Moreover, in Eqs. (1)-(8) we can put

and

$$
\theta_{\mathrm{C}}=\theta
$$

$$
\theta_{\mathrm{D}}=\theta+\Delta \quad,
$$

being $\Delta$ the phasing angle between $m_{\mathrm{C}}$ and $m_{\mathrm{D}}$. Denoted by $m_{\mathrm{A}}$ the non-rotating total mass, the kinetic energy of the system illustrated in Fig. 2(b) is provided by the following relationship

$$
E_{\mathrm{c}}=\frac{1}{2} m_{\mathrm{A}} \dot{y}^{2}+\frac{1}{2} m_{\mathrm{C}}\left(\dot{x}_{\mathrm{C}}^{2}+\dot{y}_{\mathrm{C}}^{2}\right)+\frac{1}{2} m_{D}\left(\dot{x}_{\mathrm{D}}^{2}+\dot{y}_{\mathrm{D}}^{2}\right)
$$

\subsubsection{Lagrange's equations}

By substituting Eqs. (5)-(10) in Eq. (11), we obtain the expression of the kinetic energy directly versus the two degrees of freedom (DOF) of the system $y$ and $\theta$ ( $\Delta$ is assumed to be constant, i.e. time-independent). Putting the expression of $E_{c}$ just reported in the two Lagrange's equations relative to the corresponding DOF $y$ and $\theta$

$$
\begin{gathered}
\frac{d}{d t}\left(\frac{\partial E_{c}}{\partial \dot{y}}\right)-\frac{\partial E_{c}}{\partial y}=-F_{i d} \\
\frac{d}{d t}\left(\frac{\partial E_{c}}{\partial \dot{\theta}}\right)-\frac{\partial E_{c}}{\partial \theta}=M_{\mathrm{v}}(t),
\end{gathered}
$$

the final equations of motion of the device illustrated in Fig. 2(b) are obtained. In Eq. (13) $M_{\mathrm{v}}(t)$ is the generalized moment applied to the crank $r$ (see Fig. 2(b)). Proceeding as indicated and recalling that it was fixed $m_{\mathrm{C}}=m_{\mathrm{D}}=1 / 2 m_{\mathrm{B}}=m$ ( $m$ is the value of each counter rotating mass in the system illustrated in Fig. 1(a)), Eqs. (12) and (13) can be written as

$$
\begin{gathered}
\left(2 m+m_{\mathrm{A}}\right) \ddot{y}+m r\left[\ddot{\theta} \cos \theta+\ddot{\theta} \cos (\theta+\Delta)-\dot{\theta}^{2} \sin \theta-\dot{\theta}^{2} \sin (\theta+\Delta)\right]=-F_{i d}, \\
m r[\ddot{y}(\cos \theta+\cos (\theta+\Delta))+2 r \ddot{\theta}]=M_{\mathrm{v}}(t) .
\end{gathered}
$$


Therefore, by integrating Eq. (14) it will be possible to compare the results directly with those obtained relatively to the device studied in [10], being equal, the total mass of the hull and the counter rotating mass.

\section{Increment of the propulsion thrust through elliptical gears}

\subsection{Thurst by circular gears}

The velocity $\dot{y}$ of the system illustrated in Figs. 1(a) and 2(a) essentially depends on the following four variables that can be physically changed: i) the mass $m_{\mathrm{A}}$ of the vessel (which does not include the counter rotating masses), ii) the total mass $m_{\mathrm{B}}=2 \mathrm{~m}$ of all the rotating parts, iii) the crank radius $r$, and iv) the function $\theta(t)$ (and then $\dot{\theta}(t), \ddot{\theta}(t)$ ). The parameters $m_{\mathrm{A}}$ and $m_{\mathrm{B}}$ are closely connected because the ratio $k=m_{\mathrm{A}} / m_{\mathrm{B}}$ is a fundamental quantity to be able to obtain significant displacements $y$ and velocities $\dot{y}$ of the system (for example see Fig. 12 in [10]). The mass $m_{\mathrm{A}}$ is given by the sum of the masses relative to the hull, the engine, and the whole bearing structure (excluding the counter rotating parts) of the propulsion device. We note that an increment of the mass $m_{\mathrm{B}}$ allows one to obtain higher values of the thrust which generates the forward motion of the hull. Nevertheless, there are certainly engineering constraints concerning the practical possibility to manufacture a prototype of the system to perform experimental tests, surely $r$ and $m_{\mathrm{B}}$ can not be increased beyond certain limits. Then, in order to increase the displacement of the vessel, being equal masses and geometry, the parameter that one can reasonably settle is the choice of the laws $\theta(t), \dot{\theta}(t)$ e $\ddot{\theta}(t)$. Consequently, these laws will have to be suitably chosen so as $\theta, \dot{\theta}$, and $\ddot{\theta}$ during each rotation of the counter rotating masses vary in such a way to maximize the forward thurst on the hull. In the following paragraph we describe how it is possible to obtain such a result.

\subsection{Thurst by elliptical gears}

In order to increase the propulsion thurst we can impose that the counter rotating masses do no longer rotate with a constant angular velocity $\dot{\theta}$ during the stationary working as assumed in [10]. With reference to the domain represented by each complete rotation from 0 to $2 \pi$, the new function $\dot{\theta}$ will be periodic, but non-harmonic. In order to define this function, from the transmission of motion point of view a proposal assessable is represented by the use of elliptical gears instead of circular gears. As it will be shown successively, this choice allows one to obtain higher performance in terms of velocity of the vessel, being equal the angular velocity of the driving motor of the counter rotating masses. Moreover, a higher reliability compared with the case where an electronic control of the rotation speed of the masses is used can be attained. In order to obtain the phasing angle $\Delta$ and the rotation of $m_{\mathrm{C}}$ and $m_{\mathrm{D}}$, the utilisation of two cylindrical gears meshed with an idle wheel is considered. In Fig. 3(a) these three wheels are drawn by the simple representation of the pitch circles $\mathscr{C}_{\mathrm{C}}, \mathscr{C}_{\mathrm{D}}$ and $\mathscr{C}_{\mathrm{O}}$. The circles $\mathscr{C}_{\mathrm{C}}$ and $\mathscr{C}_{\mathrm{D}}$ are those relating to the gear wheels which are keyed to the shafts that rotate the respective masses $m_{\mathrm{C}}$ and $m_{\mathrm{D}}$. The circumference $\mathscr{C}_{\mathrm{O}}$ is associated with the idle gear which imposes the same angular velocity $\dot{\theta}(t)$ to $m_{\mathrm{C}}$ and $m_{\mathrm{D}}$. In Fig. 3(b) the pitch ellipses $\mathscr{C}_{\mathrm{m}}$ and $\mathscr{C}_{\mathrm{v}}$ of two elliptic gears meshed with each other and rotating around the respective foci $\mathrm{D}$ and $\mathrm{D}^{\prime}$ are illustrated. The upper wheel is firmly keyed to the cylindrical gear shown by the pitch circle $\mathscr{C}_{\mathrm{D}}$ and the axis of rotation of this wheel passes right through the lower focus of the above-mentioned ellipse. The gear associated with the pitch ellipse $\mathscr{C}_{\mathrm{m}}$ is the driving wheel of the system and to this wheel, by the shaft keyed in D' (lower focus of $\mathscr{C}_{\text {m }}$ ), a certain 
angular velocity is imposed. In the simplest case, this velocity will define a transient startup, a stationary working (constant angular velocity), and then a stop transient.

\subsection{Qualitative prediction on the motion of the vessel by elliptical gears}

Both mechanisms illustrated in Fig. 2, if the force $F_{i d}$ is equal to zero, simply oscillate along the direction of the axis $Y$. As a matter of fact, in this case there is no reaction force that opposes the component of the centrifugal force towards the axis $Y$. Conversely, if a certain "constraint degree" of the cursor along the $Y$ axis exists, a reaction that is always opposed to the displacement along the $Y$ axis of the system cursor-rotating masses will be generated. This partial constraint is given by the water that surrounds the boat and the corresponding reaction is represented by the hydrodynamic drag force $F_{i d}$ that always opposes the displacement of the system. Considering the functioning principle of the device, studied in detail in [10], we observe that the higher the component of the centrifugal force along the positive direction of the $Y$ axis, the greater the tendency to move in that direction. This behaviour occurs because when the vessel moves forward, the modulus of $F_{i d}$ is lower than that of the backward motion. It follows that if the modulus of the centrifugal force applied to the rotating masses shown in Fig. 2 assumes a maximum and a minimum value when $0 \leq \theta \leq \pi$ and $\pi \leq \theta \leq 2 \pi$, respectively, it can be expected that the system will move forward with a greater average speed along the positive direction of the $Y$ axis with respect to the case where the modulus of the centrifugal force is constant during the whole rotation, the average angular velocity of the rotating masses being equal. From an engineering point of view this periodic change of the centrifugal force modulus can be obtained by using elliptical gears. It is observed that with this

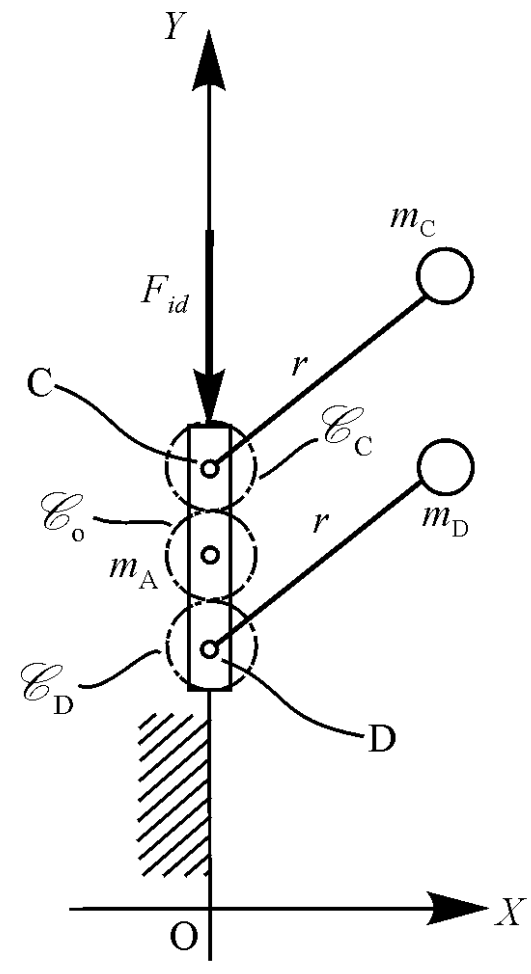

(a)

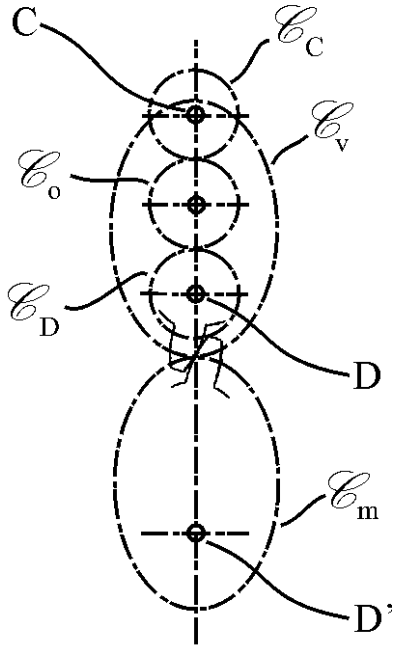

(b)

Fig. 3 (a) Train of circular gears with idle wheel to rotate and time the rotating masses $m_{\mathrm{C}}$ and $m_{\mathrm{D}}$ and (b) operation of the same train of gears by unilobe elliptical spur gears. 
solution the speed $\dot{y}$ of the boat will be characterized by oscillations whose peak values are definitely higher than those calculated in the case of the uniform circular motion of the rotating masses.

\section{Kinematic of elliptical gears}

\subsection{Polar equations of the pitch ellipses}

When the law of motion $\theta_{\mathrm{m}}(t)$ of the driving elliptical wheel is known, to determine the law of motion $\theta_{\mathrm{v}}(t)$ of the driven elliptical gear we consider the pitch ellipses $\mathscr{C}_{\mathrm{m}}$ and $\mathscr{C}_{\mathrm{v}}$ of the two corresponding gears. During the rotation these two ellipses are always tangent and roll over each other without sliding, as is the case of the pitch circles of two common circular gears. Fig. 4(a) shows a pair of pitch ellipses $\mathscr{C}_{\mathrm{m}}$ and $\mathscr{C}_{\mathrm{v}}$ and the meshing of two teeth of the corresponding cogwheels. These wheels are identical and are hinged at the points D and D' that coincide with the bottom foci of the ellipses. $a$ and $b$ denote the greater and lower semiaxis respectively of the same ellipses. The tangent point of the pitch curves is indicated by P. In order to study kinematic of the elliptical gears it is convenient to consider the polar equations of $\mathscr{C}_{\mathrm{v}}$ and $\mathscr{C}_{\mathrm{m}}$ shown in Fig. 4(a). They can be obtained through the following procedure. Observing Fig. 4(b), for the ellpise $\mathscr{C}_{\mathrm{v}}$ we consider the known relationships

and

$$
|\mathrm{GP}|+r_{\mathrm{v}}=2 a
$$

$$
|\mathrm{GP}|^{2}=|\mathrm{GH}|^{2}+|\mathrm{HP}|^{2}
$$

where $|\mathrm{GP}|$ represents the distance from the upper focus $\mathrm{G}$ and the final point $\mathrm{P}$ of the radius $r_{\mathrm{v}}$ that defines $\mathscr{C}_{\mathrm{v}}$ when the angle $\theta_{\mathrm{v}}$, measured from the axis $X_{\mathrm{v}}$, changes. Eq. (16) defines the conical ellipse, while Eq. (17) is obtained by applying Pitagora's Theorem to the triangle GHP. The axes $X_{\mathrm{v}}$ and $Y_{\mathrm{v}}$ represent a local reference system whose origin is the hinge $\mathrm{D}$, bottom focus of $\mathscr{C}_{\mathrm{v}}$ (see Figs. 4(a,b)). In order to define a clear geometrical configuration to justify Eq. (17), in Fig. 4(b) the angle $\theta_{\mathrm{v}}$ is greater than 270 degrees. Substituting in Eq. (17)

$$
|\mathrm{GH}|=|\mathrm{GD}|+r_{\mathrm{v}} \cos \theta_{\mathrm{v}}
$$

and

we have

$$
|\mathrm{HP}|=r_{\mathrm{v}} \sin \theta_{\mathrm{v}},
$$

$$
|\mathrm{GP}|=\sqrt{\left(|\mathrm{GD}|+r_{\mathrm{v}} \cos \theta_{\mathrm{v}}\right)^{2}+r_{\mathrm{v}}^{2} \sin ^{2} \theta_{\mathrm{v}}}
$$

Therefore, by Eq. (16), Eq. (20) becomes the following

$$
2 a-r_{\mathrm{v}}=\sqrt{\left(|\mathrm{GD}|+r_{\mathrm{v}} \cos \theta_{\mathrm{v}}\right)^{2}+r_{\mathrm{v}}^{2} \sin ^{2} \theta_{\mathrm{v}}}
$$

Squaring the two members of Eq. (21) and subtituting in the equation obtained

$$
|\mathrm{GD}|=2 a e \quad,
$$




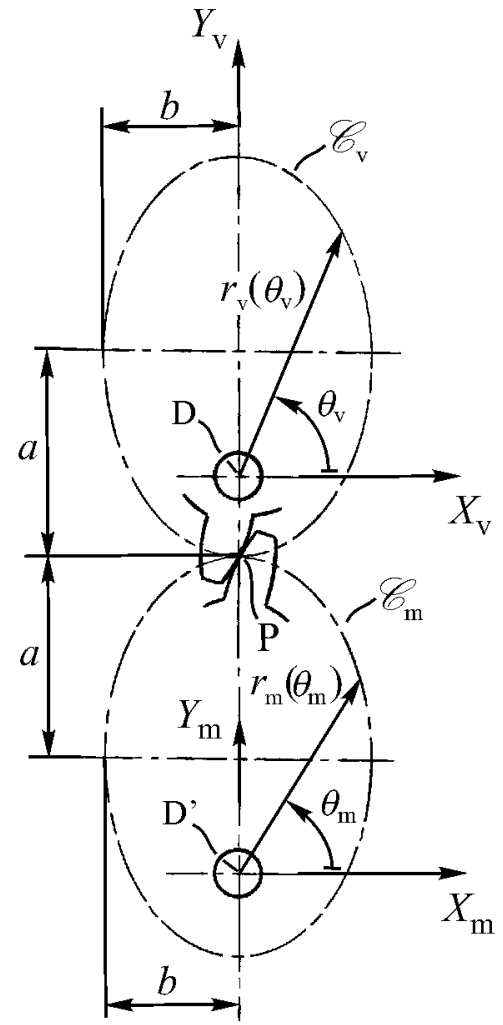

(a)

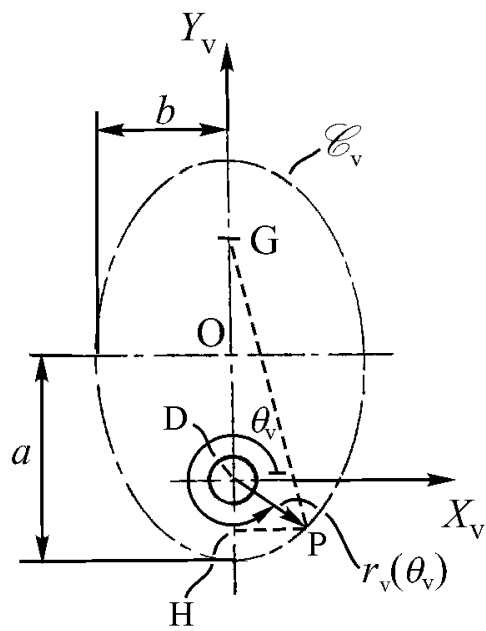

(b)

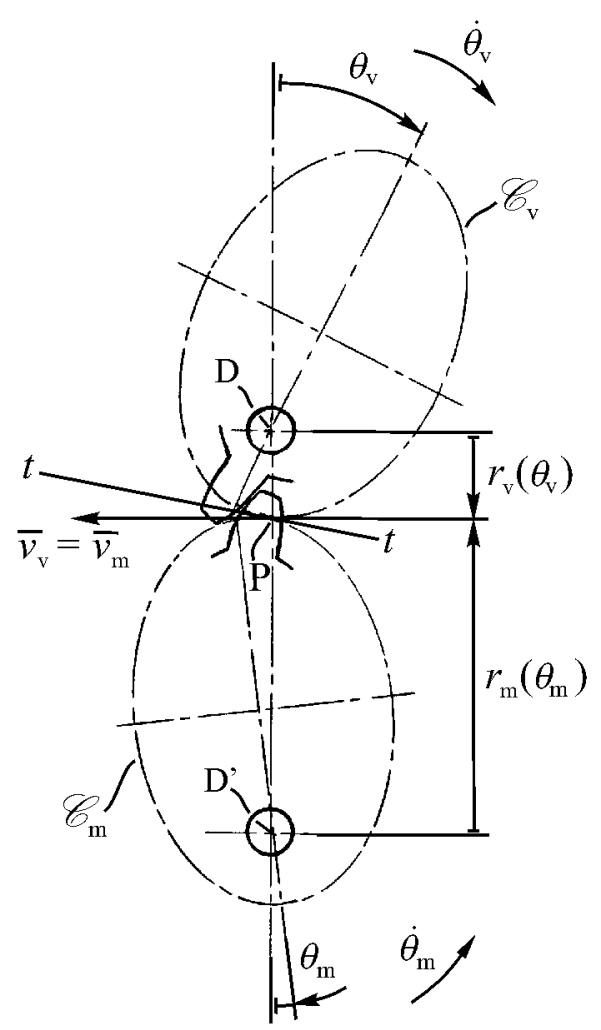

(c)

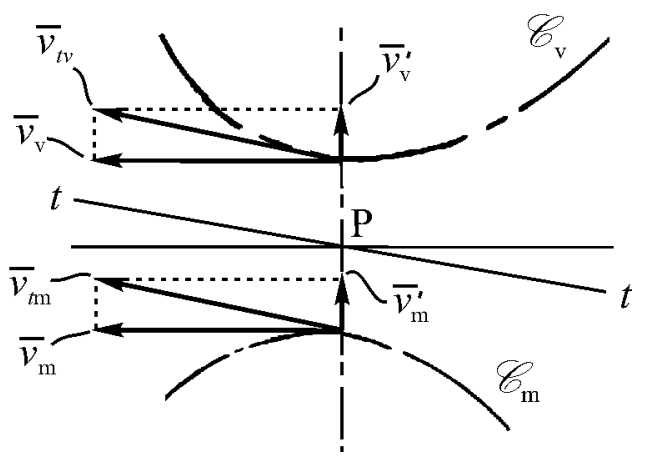

(d)

Fig. 4 Transmission of the motion by unilobe elliptical spur gears: (a) definition of the tangent pitch ellipses to each other, (b) scheme to obtain the polar equation of the pitch ellipse $\mathscr{C}_{\mathrm{v}}$, (c) rolling of the pitch ellipses, and (d) velocity in the tangency point $\mathrm{P}$ of the pitch ellipses.

where $e$ is the eccentricity of the ellipse, we draw the polar equation of $\mathscr{C}_{\mathrm{v}}$ :

$$
r_{\mathrm{v}}\left(\theta_{\mathrm{v}}\right)=\frac{a\left(1-e^{2}\right)}{1+e \cos \theta_{\mathrm{v}}}
$$


By following the same procedure we get the polar equation of $\mathscr{C}_{\mathrm{m}}$ :

$$
r_{\mathrm{m}}\left(\theta_{\mathrm{m}}\right)=\frac{a\left(1-e^{2}\right)}{1+e \cos \theta_{\mathrm{m}}}
$$

\subsection{Kinematic analysis of elliptical gears}

\subsubsection{Velocity analysis of the cogwheels contact points}

Let us consider a generic rotation of the two elliptical meshed cogwheels around D and D'. The corresponding pitch ellipses $\mathscr{C}_{\mathrm{v}}$ and $\mathscr{C}_{\mathrm{m}}$ roll without sliding and are always tangent at point $\mathrm{P}$ (see Fig. 4(c) and the tangent $t$ - $t$ passing through P). Denoting again by $\theta_{\mathrm{v}}$ the angle of rotation of $\mathscr{C}_{\mathrm{v}}$ measured, this time, from the vertical line passing through $\mathrm{P}$ as shown in Fig. 4(c), we note that the segment DP corresponds to the radius $r_{v}\left(\theta_{v}\right)$ evaluated by the relationship (23). Similarly, for the driving gear and the corresponding ellipse $\mathscr{C}_{\mathrm{m}}$ the distance D'P is equal to the radius $r_{\mathrm{m}}\left(\theta_{\mathrm{m}}\right)$ provided by Eq. (24). Since the two pitch ellipses $\mathscr{C}_{\mathrm{v}}$ and $\mathscr{C}_{\mathrm{m}}$ roll without sliding and are always tangent at $\mathrm{P}$, there is no intersection of the curves themselves. Consequently, the same thing happens with regard to the sides of the teeth meshed, at least for teeth properly configured. This condition is satisfied only when the point $\mathrm{P}$ thought to belong to $\mathscr{C}_{\mathrm{v}}$ has a velocity $\bar{v}_{t \mathrm{v}}$ equal to that $\bar{v}_{t \mathrm{~m}}$ of the same point $\mathrm{P}$ thought belonging to $\mathscr{C}_{\mathrm{m}}$ along the direction of the tangent $t$ - $t$ (see Fig. $4(\mathrm{~d}))$. Therefore it is always

$$
\bar{v}_{t v}=\bar{v}_{t \mathrm{~m}} \quad .
$$

It follows that also the horizontal $\bar{v}_{\mathrm{v}}$ and vertical $\bar{v}_{\mathrm{v}}{ }_{\mathrm{v}}$ components of $\bar{v}_{\mathrm{tv}}$ are equal to the relative horizontal $\bar{v}_{\mathrm{m}}$ and vertical $\bar{v}_{\mathrm{m}}^{\prime}$ components of $\bar{v}_{\mathrm{tm}}$ :

$$
\begin{aligned}
& \bar{v}_{\mathrm{v}}=\bar{v}_{\mathrm{m}}, \\
& \bar{v}_{\mathrm{v}}^{\prime}=\bar{v}_{\mathrm{m}}^{\prime} \quad,
\end{aligned}
$$

\subsubsection{Evaluation of the instantaneous gear ratio}

From Eq. (26) we carry out (see Fig. 4(c))

$$
\dot{\theta}_{\mathrm{v}} r_{\mathrm{v}}\left(\theta_{\mathrm{v}}\right)=\dot{\theta}_{\mathrm{m}} r_{\mathrm{m}}\left(\theta_{\mathrm{m}}\right)
$$

where $\dot{\theta}_{\mathrm{v}}$ and $\dot{\theta}_{\mathrm{m}}$ are the angular velocities by which the driven and driving wheels rotate, respectively. Substituting Eqs. (23) and (24) in Eq. (28) we obtain the following relationship

$$
\dot{\theta}_{\mathrm{v}} \frac{a\left(1-e^{2}\right)}{1+e \cos \theta_{\mathrm{v}}}=\dot{\theta}_{\mathrm{m}} \frac{a\left(1-e^{2}\right)}{1-e \cos \theta_{\mathrm{m}}}
$$

from which we obtain the angular velocity of the driven gear $\dot{\theta}_{\mathrm{v}}$ versus the velocity $\dot{\theta}_{\mathrm{m}}$ of the driving gear:

$$
\dot{\theta}_{\mathrm{v}}=\dot{\theta}_{\mathrm{m}} \frac{1+e \cos \theta_{\mathrm{v}}}{1-e \cos \theta_{\mathrm{m}}}
$$


As soon as the functions $\theta_{\mathrm{m}}(t)$ and $\dot{\theta}_{\mathrm{m}}(t)$ are fixed, Eq. (30) represents a differential equation of the first order whose unknown is $\theta_{\mathrm{v}}(t)$. This equation can be integrated over time to achieve the rotation $\theta_{\mathrm{v}}(t)$ of the driven wheel when the driving gear wheel is rotated with $\theta_{\mathrm{m}}(t)$. By Eq. (30) the instantaneous gear ratio $i$ of the elliptical wheels is defined:

$$
i=\frac{\dot{\theta}_{\mathrm{v}}}{\dot{\theta}_{\mathrm{m}}}=\frac{1+e \cos \theta_{\mathrm{v}}}{1-e \cos \theta_{\mathrm{m}}}
$$

We observe that $\theta_{\mathrm{m}}(t)$ and therefore also $\dot{\theta}_{\mathrm{m}}(t)$ are known, while $\theta_{\mathrm{v}}(t)$ and $\dot{\theta}_{\mathrm{v}}(t)$ are unknown. $\theta_{\mathrm{v}}(t)$ and $\dot{\theta}_{\mathrm{v}}(t)$, as above-mentioned, can be obtained by solving the differential equation (30). Therefore $i$ can be computed only when $\dot{\theta}_{\mathrm{v}}(t)$ (or $\theta_{\mathrm{v}}(t)$ ) are available. By observing that the distance DD' between the two rotation centres $\mathrm{D}$ and $\mathrm{D}^{\prime}$ is constant and is equal to

$$
\mathrm{DD}^{\prime}=r_{\mathrm{m}}\left(\theta_{\mathrm{m}}\right)+r_{\mathrm{v}}\left(\theta_{\mathrm{v}}\right)
$$

an alternative for the calculation of $i$ is represented by the solution of the non-linear algebraic system constituted by the two equations

$$
\begin{aligned}
\mathrm{DD}^{\prime} & =r_{\mathrm{m}}\left(\theta_{\mathrm{m}}\right)+\frac{a\left(1-e^{2}\right)}{1+e \cos \theta_{\mathrm{v}}}, \\
i & =\frac{1+e \cos \theta_{\mathrm{v}}}{1-e \cos \theta_{\mathrm{m}}},
\end{aligned}
$$

referring to each instant $t$ where $i$ has to be computed. Eq. (33) was obtained from Eq. (32) by replacing $r_{\mathrm{v}}\left(\theta_{\mathrm{v}}\right)$ with the corresponding expression given by Eq. (23). $r_{\mathrm{m}}\left(\theta_{\mathrm{m}}\right)$ is evaluated by Eq. (24) considering a fixed value of $\theta_{\mathrm{m}} \cdot \theta_{\mathrm{v}}$ and $i$ are the two unknowns of the non-linear system. So, it is possible to solve the system defined by Eqs. (33) and (34). Otherwise, with reference directly to the domain of the angle of rotation $\theta_{\mathrm{m}}$, the same angle can be varied from 0 to $2 \pi$ and, for each value of $\theta_{\mathrm{m}}$ and $r_{\mathrm{m}}\left(\theta_{\mathrm{m}}\right)$, the above-mentioned system is solved. The correspondent solution provides numerically the value of the function $i\left(\theta_{\mathrm{m}}\right)$. Anyway, this ratio is always a periodic function that repeats itself referring to each complete revolution of the driven and driving gears.

\section{Evaluation of the propulsive efficiency}

In order to calculate the propulsive efficiency of the devices equipped with one and two pairs of counter rotating masses, to know all the parameters that define the motion over time (in particular, displacement, velocity and acceleration) the equations of motion of the system must be necessarily integrated. In relation to the complexity of these equations of motion, the integrations have to be numerically carried out. However, this procedure is not always without drawbacks so, during the numerical integration, in relation to the software used [22] (Mathematica), sometimes spurious values that locally alter those correct are produced. This problem affects the derivatives obtained by numerical integration: velocity and especially acceleration (both linear and angular) sometimes show anomalous peaks that can not actually happen because they represent real discontinuities that are introduced by the numerical techniques implemented in the integration software used. These mistakes could not be removed, but to calculate the efficiency it is necessary to proceed with further integration of these functions and the anomalous peaks (discontinuities) can prevent these further calculations. In order to minimize the inconvenience just described, the specific experience 


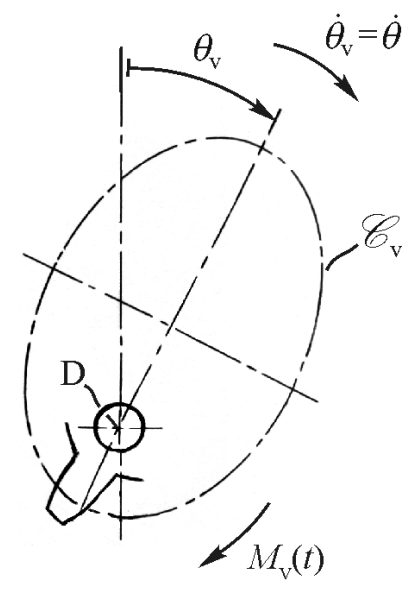

(a)

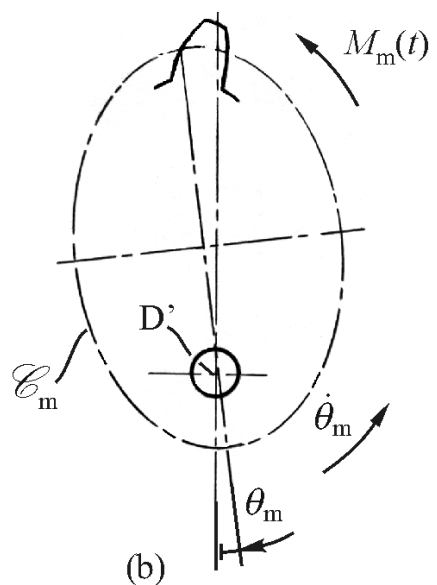

(b)

Fig. 5 (a) Driven and (b) driving cogwheel and relative moments $M_{\mathrm{V}}(t)$ and $M_{\mathrm{m}}(t)$ applied.

developed in the present work has shown that is convenient to avoid certain combinations of products or divisions of these functions that enhance too much the same discontinuities. This way it was found that by directly utilising the principle of conservation of energy to evaluate the torque that has to be applied to the driving elliptic gear, it is possible to minimize the problem previously described. Concernig this, it is observed that in the Lagrange's equation (15) the functions $\theta(t)$, $\dot{\theta}(t)$, and $\ddot{\theta}(t)$ have been fixed a priori (are equal to $\theta_{\mathrm{v}}, \dot{\theta}_{\mathrm{v}}$, and $\ddot{\theta}_{\mathrm{v}}$ respectively, see Fig. 4 ). In the real case, the masses $m_{\mathrm{C}}$ and $m_{\mathrm{D}}$ can rotate with velocity $\dot{\theta}(t)=\dot{\theta}_{\mathrm{v}}(t)$ only when a moment $M_{\mathrm{v}}(t)$ is applied to the driven elliptical gear. This gear rotates simultaneously the two cranks $r$ on which $m_{\mathrm{C}}$ and $m_{\mathrm{D}}$ are fixed. The other terms $\theta(t)$ and $\ddot{\theta}(t)$ of Eq. (15) are carried out, for example, by integrating Eq. (30). When $M_{\mathrm{v}}(t)$ has been computed, the product $M_{\mathrm{v}}(t) \dot{\theta}(t)$ provides the instantaneous power that must be supplied to the system to obtain the displacement $y(t)$. As it will be shown in the next section, this power can be used to calculate the efficiency of the propulsion system. However, performing these kinds of calculations the drawbacks previously described are found (excessive spurious values affect the functions calculated) and it is very difficult to obtain reliable results. As a matter of fact, the function $M_{\mathrm{v}}(t)$ obtained through the above-mentioned procedure shows high peaks that depend on $\ddot{y}(t)$. Since, in order to evaluate the efficiency of the propulsion system, it is necessary to integrate the power $M_{\mathrm{v}}(t) \dot{\theta}(t)$, the poor continuity of this function prevents a reliable numeric integration. On the contrary, utilising a formulation based directly on the principle of conservation of energy where the input energy to the system is evaluated with reference to the driving elliptical gear and not to the driven one (to which Eq. (15) relates), we will get good results.

\subsection{Analysis in-depth of the moments applied}

Let us consider the system illustrated in Fig. 5(a), which shows the pitch ellipses $\mathscr{C}_{\mathrm{v}}$ of the cogwheel rotating around D with angular velocity $\dot{\theta}(t)=\dot{\theta}_{\mathrm{v}}(t)$. Such cogwheel rotates because its tooth is subject to the force applied by the other meshed tooth. This force generates the moment $M_{\mathrm{v}}(t)$. Therefore, the instantaneous input power furnished to the system constitued by the coghwheel rotating around $\mathrm{D}$ and all the parts joined to it, that is the masses $m_{\mathrm{A}}, m_{\mathrm{C}}$, and $m_{\mathrm{D}}$, is 
equal to $M_{\mathrm{v}}(t) \dot{\theta}(t)$. However, since in this simplified model we don't consider the friction nor the masses of the two cogwheels together with the other various parts that define the mechanical transmission of the motion (shafts, bearings, other gears, etc.), the instantaneous input power $M_{\mathrm{v}}(t) \dot{\theta}(t)$ is equal to the instantaneous input power $M_{\mathrm{m}}(t) \dot{\theta}_{\mathrm{m}}(t)$ furnished to the cogwheel with pitch ellipses $\mathscr{C}_{\mathrm{m}}$ (see Fig. 5(b)) by the motor. Consequently, it results $M_{\mathrm{v}}(t) \dot{\theta}(t)=M_{\mathrm{m}}(t) \dot{\theta}_{m}(t)$ and we can perform a reliable integration of the function $M_{\mathrm{m}}(t) \dot{\theta}_{m}(t)$ instead of $M_{\mathrm{v}}(t) \dot{\theta}(t)$. The function $M_{\mathrm{m}}(t) \dot{\theta}_{m}(t)$ is much more continuous than $M_{\mathrm{v}}(t) \dot{\theta}(t)$ because, in order to compute the system efficiency, we consider $\dot{\theta}_{m}(t)$ to be a constant, that is $\dot{\theta}_{m}(t)=\dot{\theta}_{m}$, while $\dot{\theta}(t)=\dot{\theta}_{\mathrm{v}}(t)$ changes (it depends on the istantaneous gear ratio of the elliptical wheels).

\subsection{Definitions of efficiency}

In general, the efficiency is defined by the ratio between the energy supplied to the system by a motor to generate the motion and the energy that the system really absorbs to go forward along a fixed direction. The efficiency can be evaluated instant by instant, for example as the ratio between the instantaneous powers corresponding to a time $t$, or with reference to the average values of power calculated by considering a certain interval of time. Usually, a good indication of the propulsive efficiency is actually given with reference to the above-mentioned interval of time. This efficiency, that can be defined as an average efficiency, is given by the following relation

$$
\eta_{m p}=\frac{P_{m d}}{P_{\text {mav }}},
$$

where $P_{\text {mav }}$ is the average power given to the engine of the system and $P_{m d}$ is the average power that the same system absorbs to go forward along a predetermined direction in a certain time interval $\Delta t$, for example, with reference to a steady state working. $P_{\text {mav }}$ and $P_{m d}$ can be computed by applying the definition of average value of a function to the corresponding instantaneous power $P_{a v}(t)$ and $P_{d}(t)$ :

$$
\begin{aligned}
& P_{m a v}=\frac{1}{\Delta t} \int_{0}^{\Delta t} P_{a v}(t) d t, \\
& P_{m d}=\frac{1}{\Delta t} \int_{0}^{\Delta t} P_{d}(t) d t
\end{aligned}
$$

$P_{a v}(t)$ and $P_{d}(t)$ are given by the following equations

$$
\begin{aligned}
& P_{a v}(t)=M_{\mathrm{m}}(t) \dot{\theta}_{\mathrm{m}}(t) \\
& P_{d}(t)=F_{i d}[\dot{y}(t)] \dot{y}(t)
\end{aligned}
$$

where $M_{\mathrm{m}}(t)$ is the torque applied to the driving gear that rotates with angular velocity $\dot{\theta}_{\mathrm{m}}(t)$ at the instant $t$. 


\subsection{Equation of energy}

\subsubsection{Evaluation of the kinetic energy change}

The principle of conservation of energy applied to a mechanical system is defined by the following equation

$$
d E=d L_{\mathrm{m}}-\left(d L_{\mathrm{p}}+d L_{\mathrm{r}}\right)
$$

where $d E$ is the infinitesimal variation of kinetic energy of the system. Analogously, $d L_{\mathrm{m}}, d L_{\mathrm{p}}$, and $d L_{\mathrm{r}}$ are infinitesimal variations of work: $d L_{\mathrm{m}}$ is furnished to the system, $d L_{\mathrm{p}}$ and $d L_{\mathrm{r}}$ are works generated by the friction and external load forces applied to the same system, respectively. Eq. (40) represents the energy equation of a machine and the terms $d E, d L_{\mathrm{m}}, d L_{\mathrm{p}}$, and $d L_{\mathrm{r}}$ referring to a certain reference axis. The choice of this axis is performed in such a way as to simplify as much as possible the expression of the above-mentioned terms. For the sake of simplicity we can neglect $d L_{\mathrm{p}}$ that represents the work done by the friction forces between the parts in relative motion of the driving mechanism of the counter rotating masses (friction in the bearings, friction between the teeth of the gears meshed, etc.). The infinitesimal variation of kinetic energy $d E$ is given by the following relationship

$$
d E=d\left(\frac{1}{2} m_{t o t} \dot{y}^{2}+\frac{1}{2} I \dot{\theta}_{\mathrm{m}}^{2}\right)
$$

where $m_{\text {tot }}$ is the total mass of the system that translates with velocity $\dot{y}$ and $I$ is the reduced mass moment of inertia with respect to the reference axis previously chosen. If we consider only the stationary working of the propulsion system, the shaft that rotates with constant angular velocity is the one on which is keyed the driving elliptical gear. Then, it is convenient to choose precisely the axis of this shaft as the reference axis.

\subsubsection{Evaluation of the works}

The infinitesimal work $d L_{\mathrm{m}}$ furnished to the system is equal to

$$
d L_{\mathrm{m}}=M_{\mathrm{m}} d \theta_{\mathrm{m}},
$$

where $M_{\mathrm{m}}$ is the torque applied to the driving gear and $d \theta_{\mathrm{m}}$ is the corresponding infinitesimal rotation of the shaft on which the same gear is keyed. $d L_{\mathrm{r}}$ represents the infinitesimal work of the load forces applied to the system. In the case study is

$$
d L_{\mathrm{r}}=F_{i d} d y \quad,
$$

where $F_{i d}(\dot{y})$ is the force of the hydrodynamic drag that contrasts with the motion of the vessel along $y$ direction.

\subsubsection{Evaluation of the moments}

Substituting Eqs. (41)-(43) in Eq. (40), with $d L_{\mathrm{p}}=0$, we obtain the equation 


$$
d\left(\frac{1}{2} m_{t o t} \dot{y}^{2}+\frac{1}{2} I \dot{\theta}_{\mathrm{m}}^{2}\right)=M_{\mathrm{m}} d \theta_{\mathrm{m}}-F_{i d} d y
$$

that divided by $d t$ becomes the following

$$
\frac{d}{d t}\left(\frac{1}{2} m_{t o t} \dot{y}^{2}+\frac{1}{2} I \dot{\theta}_{\mathrm{m}}{ }^{2}\right)=M_{\mathrm{m}} \dot{\theta}_{\mathrm{m}}-F_{i d} \dot{y},
$$

where $\dot{\theta}_{\mathrm{m}}$, the angular velocity of the shaft that drive the system. By performing the derivative with respect to time of the term between the round brackets in the first member of the above equation, we carry out the relationship from which you can obtain the torque $M_{\mathrm{m}}$ that has to be applied to the driving gear of the system in such a way as to rotate the driven gear with an angular velocity $\dot{\theta}_{\mathrm{v}}(t)$ :

$$
M_{\mathrm{m}}=\frac{m_{t o t} \ddot{y} \ddot{y}+I \dot{\theta}_{\mathrm{m}} \ddot{\theta}_{\mathrm{m}}+F_{i d} \dot{y}}{\dot{\theta}_{\mathrm{m}}} .
$$

The above-mentioned gear is keyed on the shaft whose axis was chosen as reference to define the terms of Eq. (40). If we consider the steady working of the system where $\dot{\theta}_{\mathrm{m}}$ is constant, then $\ddot{\theta}_{\mathrm{m}}$ is equal to zero and Eq. (46) reduces to the following:

$$
M_{\mathrm{m}}=\frac{m_{t o t} \dot{y} \ddot{y}+F_{i d} \dot{y}}{\dot{\theta}_{\mathrm{m}}} .
$$

Since in Eq. (47) $\dot{\theta}_{\mathrm{m}}$ is a constant, the torque $M_{\mathrm{m}}$ depends on the product $\ddot{y} \ddot{y}$ and $\dot{y}$. From a numerical point of view, this fact allows one to obtain a function $M_{\mathrm{m}}$ that does not contain spurious values (i.e. peaks of discontinuity) such as to prevent its use for the calculation of the average power $P_{\text {mav }}$ supplied by the motor of the system.

\section{Utilization of the mathematical phisical models}

By performing the integration of the motion equations relative to the two systems illustrated in Fig. 2, being equal i) the hydrodinamic drag force $F_{i d}$ versus the velocity of translation $\dot{y}$ of the hull, ii) the initial conditions, iii) the mass of the hull, and iv) the total counter rotating mass, it is possible to evaluate and compare consistently the performances of the two propulsion systems study. Therefore, based on the knowledge of the velocity $\dot{y}$ versus time $t$ relative to a certain time domain $\Delta t$, by Eqs. (36), (37), and (35), we can calculate the average power $P_{m a v}$ consumed by the

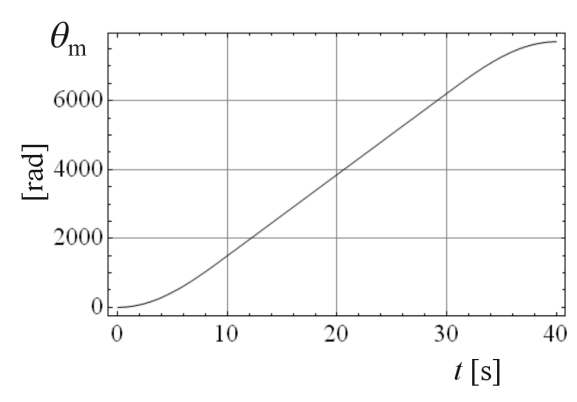

(a)

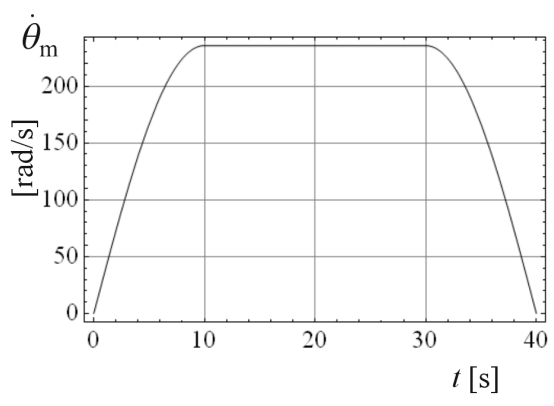

(b)

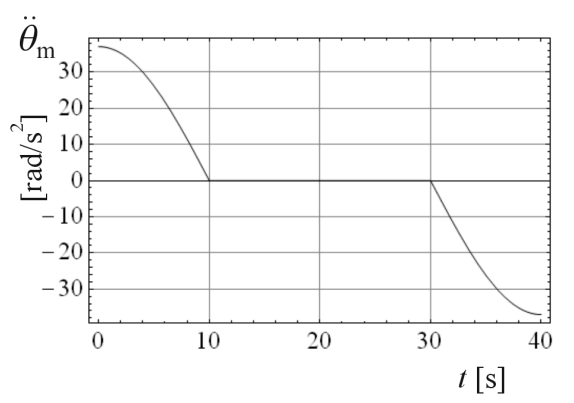

(c)

Fig. 6(a) rotation $\theta_{\mathrm{m}}(t)$, (b) angular velocity $\dot{\theta}_{\mathrm{m}}(t)$, and (c) angular acceleration $\ddot{\theta}_{\mathrm{m}}(t)$ of the driving shaft of the propulsion system. 


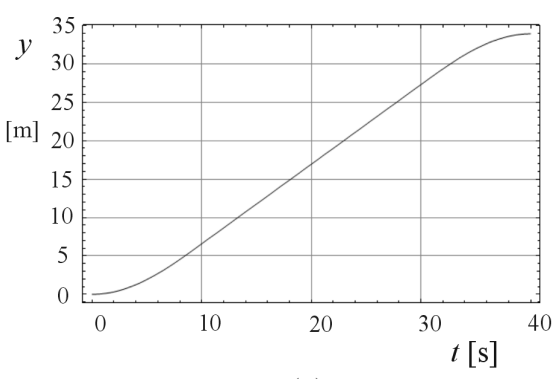

(a)

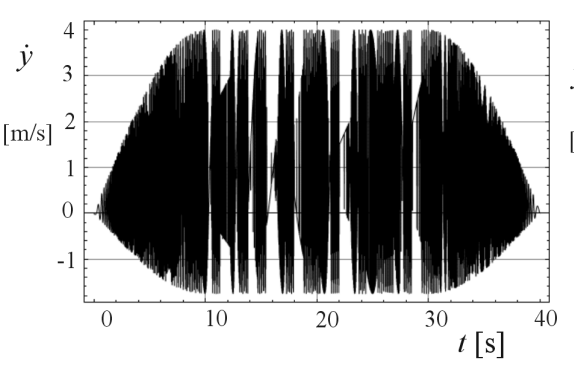

(b)

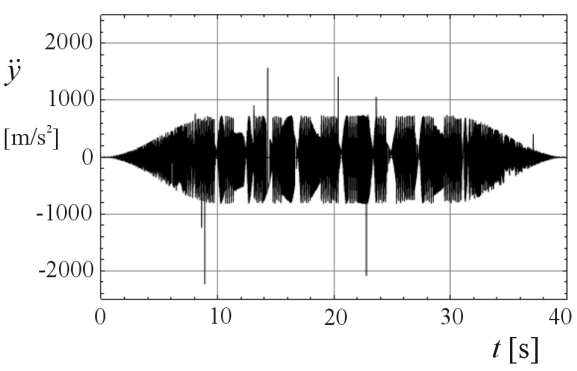

(c)

Fig. 7(a) displacement $y(t)$, (b) velocity $\dot{y}(t)$, and (c) acceleration $\ddot{y}(t)$ versus time for the partially submerged boat-like body with propulsion system constituted by only one pair of counter rotating masses.

engine of the system, the average power $P_{m d}$ that the same system absorbs to move forward along a predetermined direction, and the corresponding propulsive efficiency $\eta_{m p}$ relating to the abovementioned $\Delta t$. In the following section the results obtained according to the procedure previously illustrated will be discussed in detail. All calculations have been performed by using the software Mathematica [22].

\section{Integration of the equations of motion and results}

\subsection{Excitation functions of the system}

In order to assess whether the device equipped with two pairs of counter rotating masses has better performance in relation to the device constituted by a single pair of masses, the equations of motion of the two systems have been integrated. In particular, to assure that the comparison is correct, with the same values relative to some parameters both of the systems were fixed. These parameters are: i) the functions $\theta_{\mathrm{m}}(t), \dot{\theta}_{\mathrm{m}}(t)$, and $\ddot{\theta}_{\mathrm{m}}(t)$ that characterize the motion of the shaft which rotates the masses, ii) the hydrodinamic drag force $F_{i d}(\dot{y})$, iii) the rotation radius $r$ of the counter rotating masses, iv) the steady angular velocity $\dot{\theta}_{\mathrm{m}}$ of the above-mentioned shaft, $\left.\mathrm{v}\right)$ the total counter rotating mass $m$, and vi) the total non-counter rotating mass $m_{\mathrm{A}}$ of the system (that is the mass of the hull, of the gears support, etc.).

Table 1. Numerical values for performing the integration of the motion equations relative to the two systems illustrated in Fig. 1.

\begin{tabular}{|c|c|c|c|c|}
\hline \multirow{2}{*}{$\begin{array}{c}r \\
(\mathrm{~m})\end{array}$} & \multirow{2}{*}{$\begin{array}{c}m \\
(\mathrm{Kg})\end{array}$} & $\begin{array}{l}m_{\mathrm{A}} \\
(\mathrm{Kg})\end{array}$ & \multicolumn{2}{|c|}{$\begin{array}{c}\text { Value of } \dot{\theta}_{\mathrm{m}} \text { for the steady state } \\
\text { working }\end{array}$} \\
\cline { 4 - 5 } & & & $(\mathrm{rad} / \mathrm{s})$ & $(\mathrm{rpm})$ \\
\hline 0.090 & 2.0 & 20.000 & 235.619 & 2250.000 \\
\hline
\end{tabular}

Table 1 shows the numerical values of the parameters $r, \dot{\theta}_{\mathrm{m}}, m$, and $m_{\mathrm{A}}$ that have been fixed to compare the performances of the two propulsion system. Fig. 6 reports the functions $\theta_{\mathrm{m}}(t), \dot{\theta}_{\mathrm{m}}(t)$, and $\ddot{\theta}_{\mathrm{m}}(t)$ that have been used. These functions consider a startup transient, a steady state working, and a stopping transient, relative to a time domain equal to $40 \mathrm{~s}$. 
7.2 Integration of the motion equations

With reference to the previous functions $\theta_{\mathrm{m}}(t), \dot{\theta}_{\mathrm{m}}(t)$, and $\ddot{\theta}_{\mathrm{m}}(t)$, the motion equation of the system equipped with only one pair of counter rotating masses has been integrated by using the data reported in Table 1 and putting $\theta(t)=\theta_{\mathrm{m}}(t), \dot{\theta}(t)=\dot{\theta}_{\mathrm{m}}(t)$, and $\ddot{\theta}(t)=\ddot{\theta}_{\mathrm{m}}(t)$. The responses obtained $y(t), \dot{y}(t)$, and $\ddot{y}(t)$ are illustrated in Fig. 7. In this case we notice that the rotation of the mass $m_{\mathrm{B}}$ is simply generated by the shaft where the crank $r$ is keyed. Therefore, it results $\theta(t)=\theta_{\mathrm{m}}(t)=\theta_{\mathrm{v}}(t)$, $\dot{\theta}(t)=\dot{\theta}_{\mathrm{m}}(t)=\dot{\theta}_{\mathrm{v}}(t)$, and $\ddot{\theta}(t)=\ddot{\theta}_{\mathrm{m}}(t)=\ddot{\theta}_{\mathrm{v}}(t)$, i.e. no driven gear exists, there is only a rotating mass $m_{\mathrm{B}}$. By examining the curve reported in Fig. 7(a) it is noted that the vessel equipped with only one pair of counter rotating masses covers about $35 \mathrm{~m}$ in $40 \mathrm{~s}$. The average velocity relative to the steady period from 10 to $30 \mathrm{~s}$ corresponds to $1.03 \mathrm{~m} / \mathrm{s}(3.73 \mathrm{Km} / \mathrm{h})$. Now, let us consider the case of the propulsion system equipped with two pairs of counter rotating masses. Assuming we utilise two elliptical gears meshed with each other, we fix the transverse and conjugate diameters of the pitch ellipses $2 \mathrm{a}=0.144 \mathrm{~m}$ and $2 \mathrm{~b}=0.128 \mathrm{~m}$, respectively. These values have been chosen so that the gear can be easily housed in a hull whose maximum dimensions are the ones reported in [10]. By integrating Eq. (30) where the functions $\theta(t)=\theta_{\mathrm{m}}(t)$ and $\dot{\theta}(t)=\dot{\theta}_{\mathrm{m}}(t)$ are assumed known, the solutions $\theta_{\mathrm{v}}(t), \dot{\theta}_{\mathrm{v}}(t)$, and $\ddot{\theta}_{\mathrm{v}}(t)$ illustrated in Fig. 8 are obtained.

\subsubsection{Analysis of the impulsive response}

It is noted that the driven elliptical gear gets high peaks of angular velocity to which correspond similar angular accelerations. When we consider the steady state operation characterized by constant angular velocity $\dot{\theta}_{\mathrm{m}}(=235.6 \mathrm{rad} / \mathrm{s})$, these functions are of periodic type. With reference to the interval of time from 20.0 to 20.1 s, in Figs. 9(a), (b), and (c) such trends are shown. In these figures the periodic and impulsive trends are well highlighted against a constant trend of $\dot{\theta}_{\mathrm{m}}(t)$. Fig. 9(d) illustrates the instantaneous gear ratio, calculated by considering the ratio $\dot{\theta}_{\mathrm{v}}(t) / \dot{\theta}_{\mathrm{m}}(t)$. As soon as the functions $\theta_{\mathrm{v}}(t), \dot{\theta}_{\mathrm{v}}(t)$, and $\ddot{\theta}_{\mathrm{v}}(t)$ have been evaluated, the integration of the equation of motion (14) was carried out. In this case, it is noted that the functions $\theta(t), \dot{\theta}(t)$, and $\ddot{\theta}(t)$ are right $\theta_{\mathrm{v}}(t), \quad \dot{\theta}_{\mathrm{v}}(t)$, and $\ddot{\theta}_{\mathrm{v}}(t)$, that is $\theta(t)=\theta_{\mathrm{v}}(t), \dot{\theta}(t)=\dot{\theta}_{\mathrm{v}}(t)$, and $\ddot{\theta}(t)=\ddot{\theta}_{\mathrm{v}}(t)$. In Eq. (14) we put

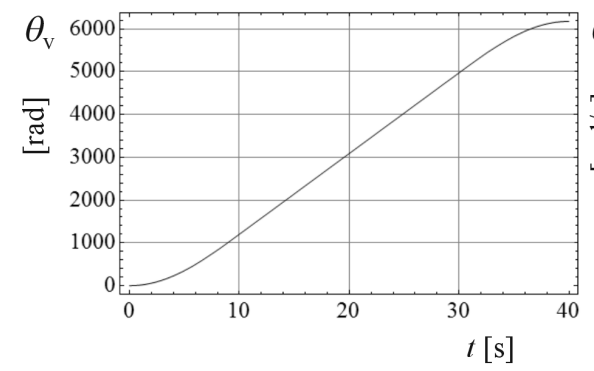

(a)

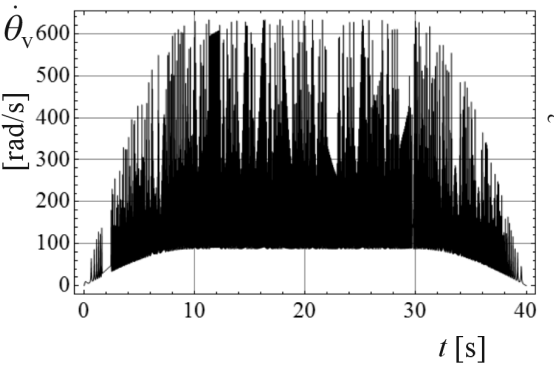

(b)

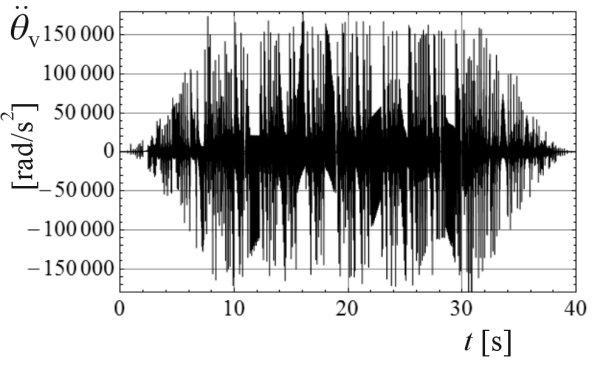

(c)

Fig. 8(a) rotation $\theta_{\mathrm{v}}(t)$, (b) angular velocity $\dot{\theta}_{\mathrm{v}}(t)$, and (c) angular acceleration $\ddot{\theta}_{\mathrm{v}}(t)$ versus time for the driven elliptical gear of the propulsion system constituted by two pairs of counter rotating masses. 
$\theta(t)=\theta_{\mathrm{v}}(t)$, because $\theta(t)$ is fixed a priori. In Fig. 10 the results of the numerical integration of Eq. (14) are reported. By using i) the data indicated in Table 1, ii) the functions $\theta_{\mathrm{v}}(t), \dot{\theta}_{\mathrm{v}}(t)$, and $\ddot{\theta}_{\mathrm{v}}(t)$ illustrated in Fig. 8, iii) the hydrodynamic drag force $F_{i d}(\dot{y})$ reported in [10], and iv) a value of the phasing angle $\Delta$ between the masses $m_{\mathrm{C}}$ and $m_{\mathrm{D}}$ equal to -5.2 degree $(-0.091 \mathrm{rad})$, we carry out the

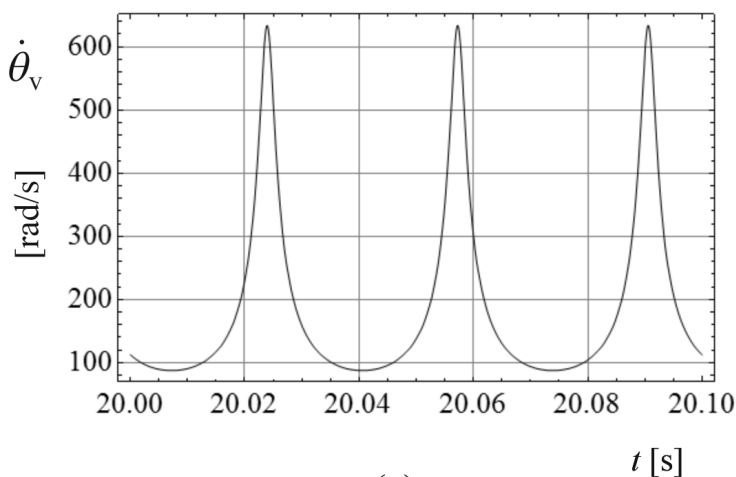

(a)

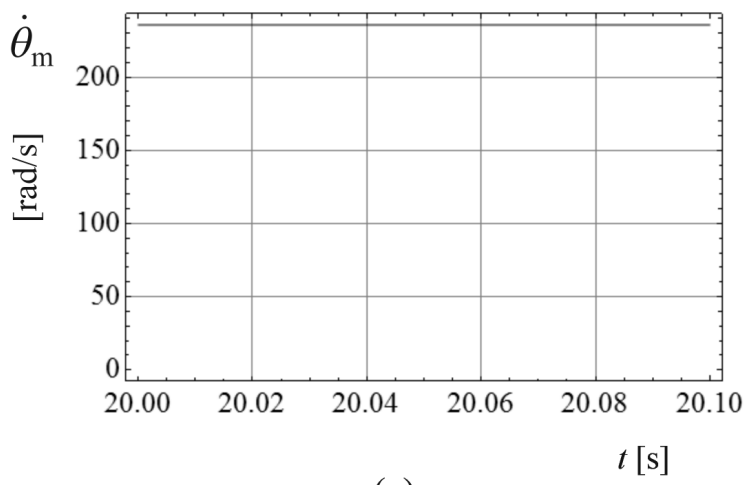

(c)

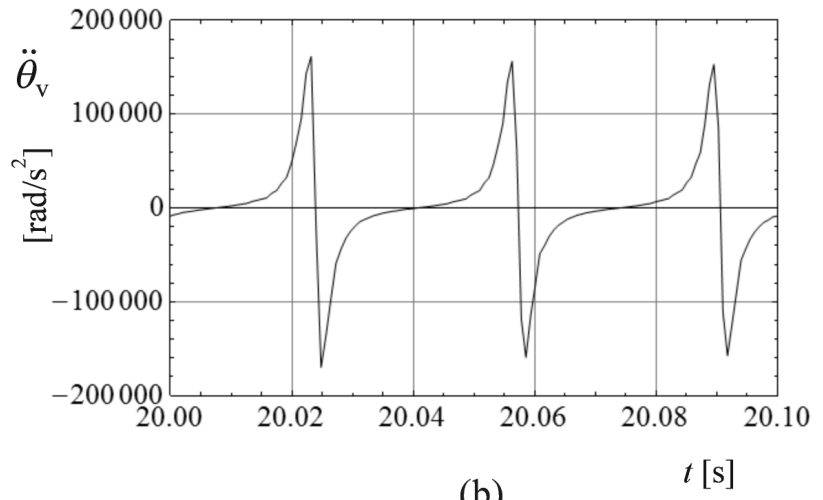

(b)

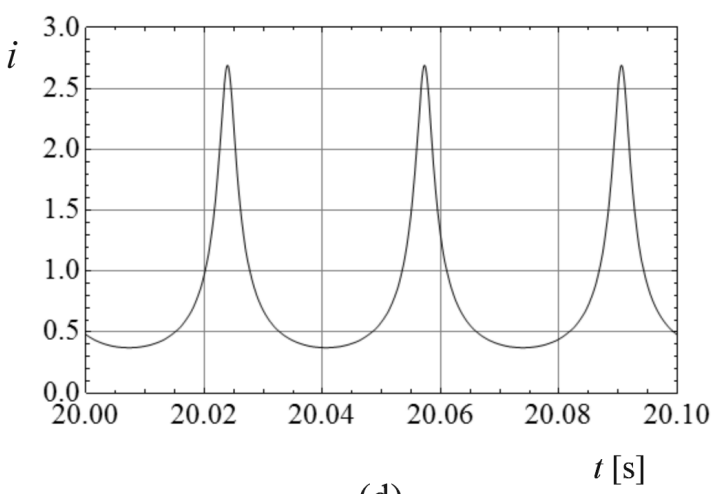

(d)

Fig. 9(a) angular velocity $\dot{\theta}_{\mathrm{v}}(t)$ and (b) angular acceleration $\ddot{\theta}_{\mathrm{v}}(t)$ for the driven elliptical gear, (c) angular velocity $\dot{\theta}_{\mathrm{m}}(t)$ of the driving elliptical gear, (d) instantaneous gear ratio $i$ of the elliptical gears meshed in the time interval from 20.0 to $20.1 \mathrm{~s}$ (steady state).

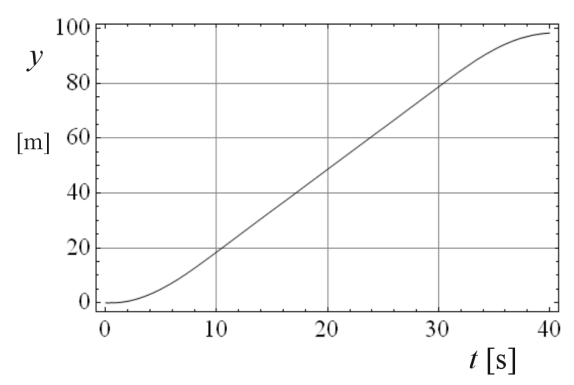

(a)

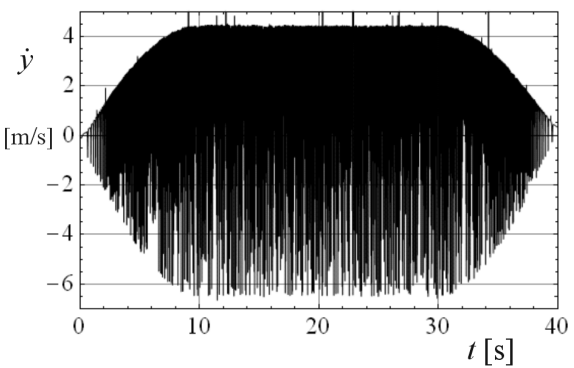

(b)

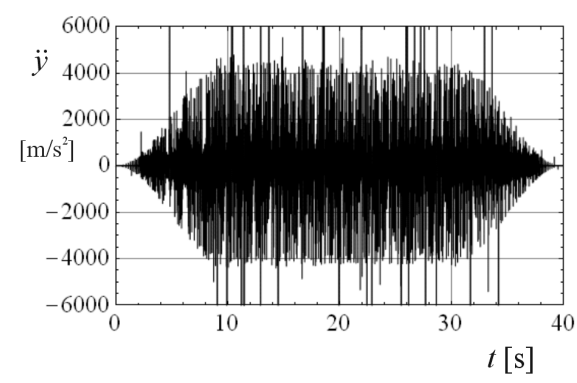

(c)

Fig. 10(a) displacement $y(t)$, (b) velocity $\dot{y}(t)$, and (c) acceleration $\ddot{y}(t)$ versus time for the partially submerged boat-like body with propulsion system constituted by two pairs of counter rotating masses and elliptical gears. 
responses $y(t), \dot{y}(t)$, and $\ddot{y}(t)$ represented in Figs. 10(a), (b), and (c), respectivley.

\subsection{Systems with one and two pairs of counter rotating masses: comparison of the results}

Comparing the aforementioned curve $y(t)$ with that obtained for the system equipped with one pair of counter rotating masses, we notice that, being equal the masses $m, m_{\mathrm{A}}$, and the angular velocity $\dot{\theta}_{\mathrm{m}}(t)$ of the motor shaft which rotates the driving gear, the distance travelled by the vessel equipped with two pairs of counter rotating masses is more than $100 \%$ higher with respect to the previous case: in $40 \mathrm{~s}$ almost $100 \mathrm{~m}$ are covered. Therefore, by the new device and the new distribution of counter rotating masses, the forward average velocity of the vessel, with reference to the steady working from 10 to $30 \mathrm{~s}$, increases from $1.03 \mathrm{~m} / \mathrm{s}(3.73 \mathrm{~km} / \mathrm{h})$ to $3.01 \mathrm{~m} / \mathrm{s}(10.82 \mathrm{~km} / \mathrm{h})$. In the latter case it is observed that the peaks of acceleration $\ddot{y}(t)$ and velocity $\dot{y}(t)$ are higher than those that characterize the system equipped with a single pair of counter rotating masses. Also the displacements are significantly different: Figs. 11(a) and (b) show the functions $y(t)$ relative to the system equipped with only one pair and two pairs of counter rotating masses respectively, with reference to the time interval from 20.0 to $20.1 \mathrm{~s}$ (case of steady working). By also observing the corresponding graphs of the velocities $\dot{y}(t)$ (see Figs. 11(c) and (d)), we can justify the fact that the function $\dot{y}(t)$ on the interval studied from 0 to $40 \mathrm{~s}$ and relative to the propulsion system equipped with elliptical gears shows peaks of negative velocity and therefore a clear prevalence of backward impulses with respect to the forward displacements of the hull. So, at a first glance, this velocity $\dot{y}(t)$ would seem in contrast with the corresponding displacement $y(t)$ of the system which shows a constant forward displacement of the boat versus time. Nevertheless, comparing the two functions $\dot{y}(t)$ shown in Figs. 11(c) and (d), it is clearly found that the time intervals where the backward displacements $(\dot{y}<0)$ happen are much shorter than those that are found in the forward displacements $(\dot{y}>0)$. The grey color of the areas under the curves $\dot{y}(t)$ shown in these figures indicates the positive values of velocity. Then, high negative peaks of velocity are defined, but their time length is very short. Consequently, a very small backward displacement of the hull happens: the forward displacement prevails because the positive velocities, also if their moduli are lower than those of the negative ones, persist for a longer time. The situation is different when we consider the propulsion device equipped with a single pair of counter rotating masses. Fig. 11(c) shows a trend of $\dot{y}(t)$ characterized by a high prevalence of positive values with respect to the negative ones, a harmonic trend is almost defined. It follows that the representation of $\dot{y}(t)$ on the complete time interval from 0 to $40 \mathrm{~s}$ indicates a high prevalence of positive values of velocity. Nevertheless, in relation to the protraction of relatively high negative velocities due to the almost harmonic shape of the same function, the entity of the forward displacement is reduced in a way much more pronounced with respect to the case of the system equipped with elliptical gears and two pairs of counter rotating masses. In this way the better performance in terms of displacement of the abovementioned propulsion system is justified. Concerning the value of the phasing angle $\Delta=-5.2$ degree $(-0.091 \mathrm{rad})$ between the two masses $m_{\mathrm{C}}$ and $m_{\mathrm{D}}$ that allows one to obtain the performance aforementioned, it was carried out by executing a set of numerical simulation. At the beginning $\Delta$ was changed from 0 to 360 degree $(2 \pi \mathrm{rad})$ by a step equal to 10 degree $(0.175 \mathrm{rad})$. The graph illustrated in Fig. 12 summarizes the results obtained and shows the total displacement $y_{t 40}$ that the vessel covers relatively to each value of $\Delta$ which changes from from 0 to $40 \mathrm{~s}$. By observing the graph we note that $y_{t 40}$ take the highest values more or less when $\Delta=0$ and $\Delta=2 \pi \operatorname{rad}$. 


\subsection{Pysical interpretation of the results}

From a physical point of view it is possible to explain why near $\Delta=0$ and $\Delta=2 \pi \mathrm{rad}$ we obtain the highest value of displacement. The functioning of the propulsion device is based on the excitation forces represented by the components along the axis $Y$ of the centrifugal forces. In Fig. 13(a) the configuration of the system when $\Delta=0$ is shown: the two centrifugal forces $F_{c \mathrm{C}}$ and $F_{c \mathrm{D}}$ applied to the corresponding masses $m_{\mathrm{C}}$ and $m_{\mathrm{D}}$ are always parallel to each other, whatever the value of $\theta$. Consequently, the resultant $R_{y}(t)$ of the relative components $F_{c \mathrm{Cy}}(t)$ and $F_{c \mathrm{Dy}}(t)$ along the axis $Y$ have the same sign at each istant $t$ and they add up. Therefore $R_{y}(t)$ is a periodic function on the domain $0 \leq \theta \leq 2 \pi$ and is defined by a certain amplitude. Conversely, when $\Delta=\pi \operatorname{rad} F_{c \text { Dy }}(t)$ assumes an opposite direction compared with the case where $\Delta=0$ (see Fig. 13(b)). Since the moduli of $F_{c \mathrm{Cy}}(t)$ and $F_{c \mathrm{Dy}}(t)$ are always equal to each other $\left(F_{c \mathrm{D}}\right.$ is $\pi \mathrm{rad}$ out of phase with respect to $\left.F_{c \mathrm{C}}\right)$, $R_{y}(t)$ is always equal to zero and no resultant force is applied to the hull to cause its oscillation along the axis $Y$. As a consequence the vessel cannot move, whatever the value of the angular speed $\dot{\theta}(t)$ of $m_{\mathrm{C}}$ and $m_{\mathrm{D}}$. On the contrary, if $\Delta=0$ (see Fig. 13(a)) we observe that $R_{y}(t)$ is equal to zero only when $\theta=0$ and $\theta=\pi \mathrm{rad}$ : for all the other values of $\theta, R_{y}(t)$ is different from zero and, as previously observed, on the domain $0 \leq \theta \leq 2 \pi$, is a periodic function. Moreover we note that only when $\Delta=0$ the amplitude of this $R_{y}(t)$ is maximum because, on the same $t$, the moduli of $F_{c \mathrm{Cy}}(t)$ and $F_{c \text { Dy }}(t)$ have always the same value and sign. Therefore, when $\Delta=0$, the maximum excitation is applied to the hull. Consequently it oscillates along $Y$ with the maximum amplitude and high

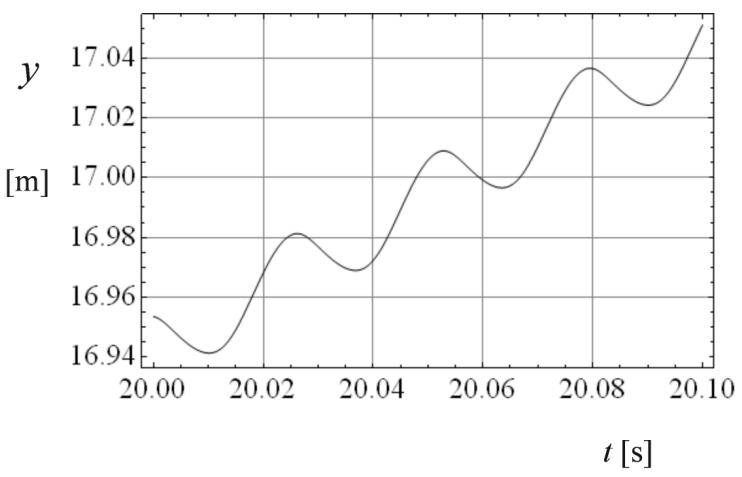

(a)

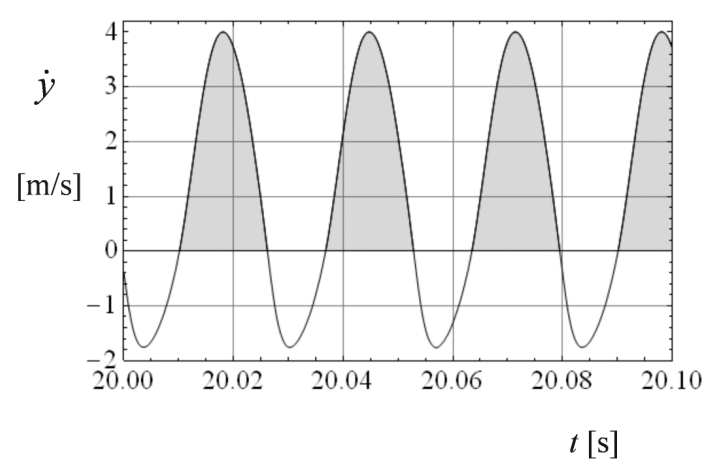

(c)

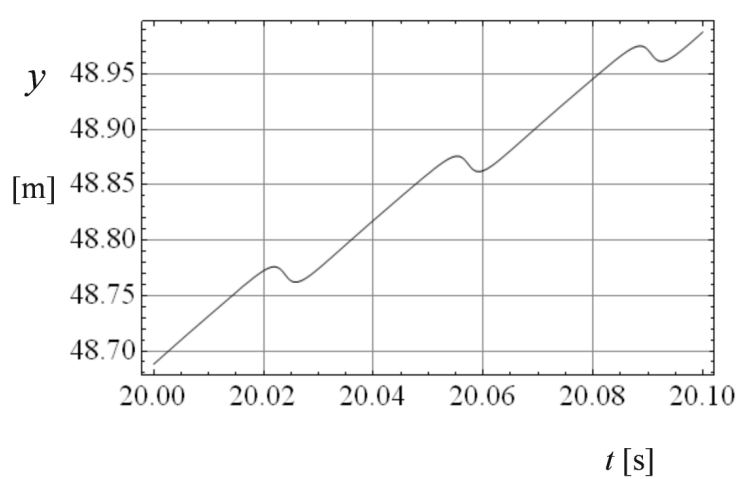

(b)

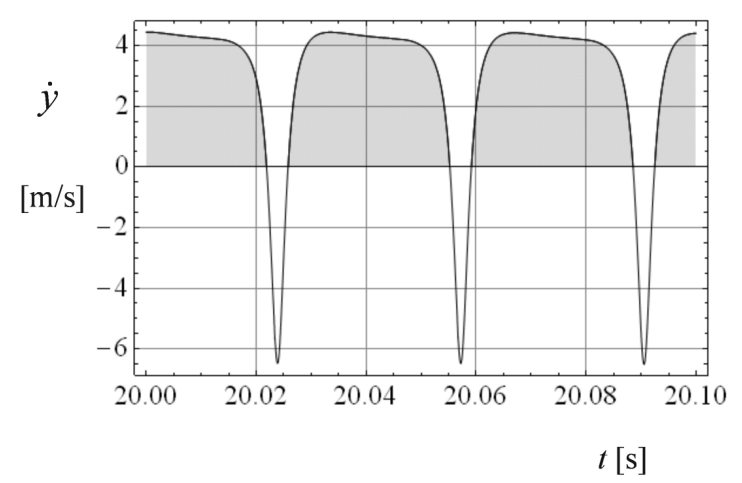

(d)

Fig. 11 Displacement $y(t)$ of the partially submerged boat-like body with propulsion system constituted (a) by only one pair and (b) two pairs of counter rotating masses; (c),(d) corresponding velocity $\dot{y}(t)$, parameters and conditions being equal, with $20.0 \leq t \leq 20.1 \mathrm{~s}$. 


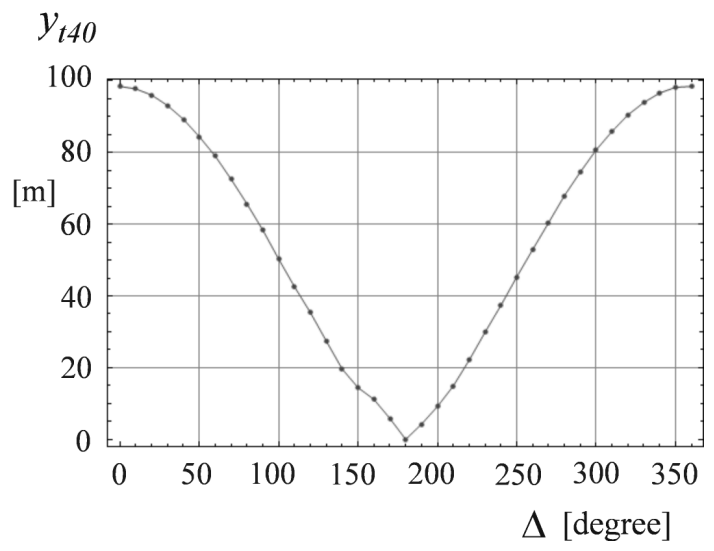

Fig. 12 Displacement $y_{t 40}$ of the partially submerged boat-like body with propulsion system constituted by two pairs of counter rotating masses and elliptical gears versus the phasing angle $0 \leq \Delta \leq 2 \pi$ rad between the masses $m_{\mathrm{C}}$ and $m_{\mathrm{D}}$.

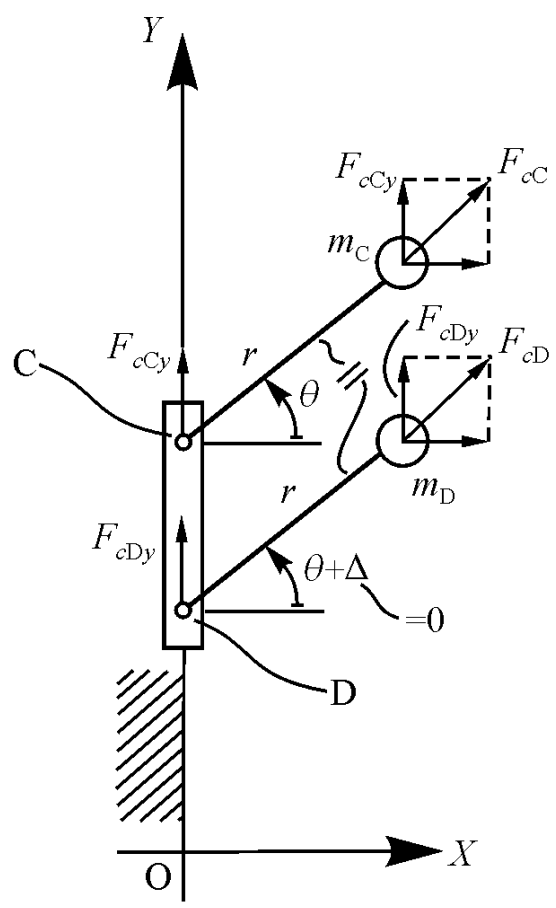

(a)

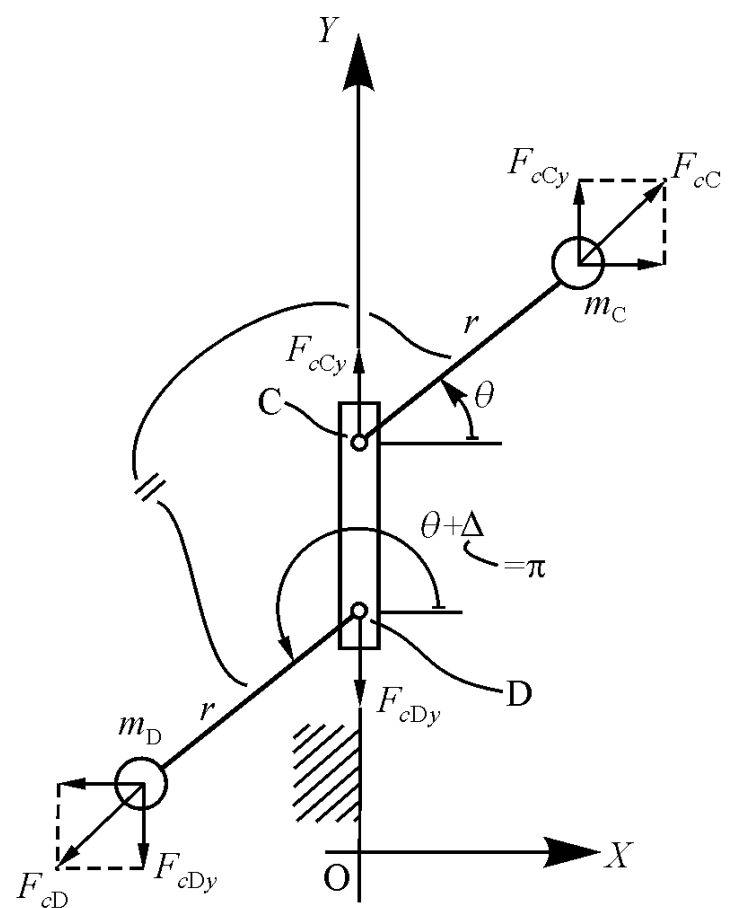

(b)

Fig. 13 Centrifugal forces $F_{c C}$ and $F_{c D}$ (a) phased ( $\left.\Delta=0\right)$ and (b) out of phase ( $\Delta=\pi \mathrm{rad}$ ).

impulsive forces move it along the axis $Y$ : the maximum value of $y_{t 40}$ is obtained. Then, motion equation (14) has been integrated by fixing a very short incremental step to $\Delta$. In particular, with $\Delta$ changing from 354 degree $(6.178 \mathrm{rad})$ to 358 degree $(6.248 \mathrm{rad})$, by using a step equal to 0.1 degree $\left(1.745 \times 10^{-3} \mathrm{rad}\right)$, the results shown in Fig. 14 were obtained. The abscissa of the graph illustrated in this figure indicates the values of $\Delta$ considered, in the ordinate the corresponding values of $y_{t 40}$ are reported. It should be noted that when $\Delta=357.8$ degree (or 360-357.8 $=-5.2$ degree $=-0.091 \mathrm{rad}$ ) we carry out the maximum displacement of the system equal almost to $100 \mathrm{~m}$, in $40 \mathrm{~s}$. 


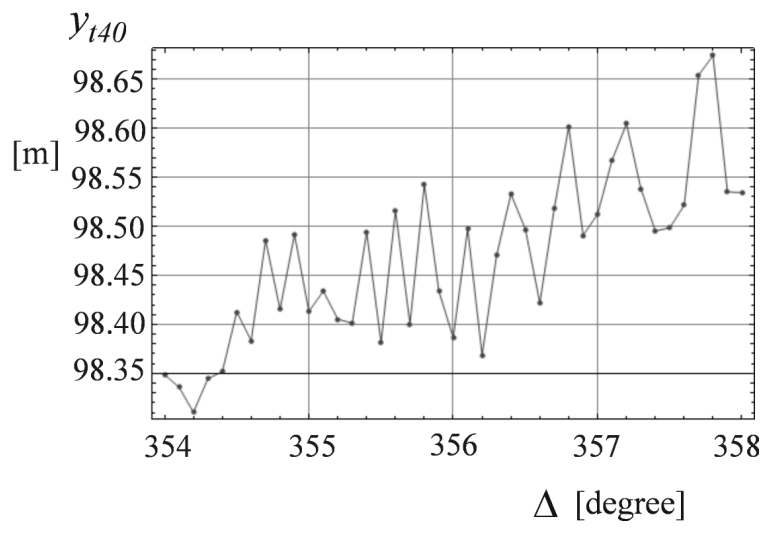

Fig. 14 Displacement $y_{t 40}$ of the partially submerged boat-like body with propulsion system constituted by two pairs of counter rotating masses and elliptical gears versus the phasing angle $6.178 \leq \Delta \leq 6.248 \mathrm{rad}$

(354 $\leq \Delta \leq 358$ degree) between the masses $m_{\mathrm{C}}$ and $m_{\mathrm{D}}$.

\section{Evaluation of the propulsive efficiency and results}

8.1 Computation of the torque $M_{\mathrm{m}}(t)$ for obtaining the motion laws $\theta_{\mathrm{v}}(t), \dot{\theta}_{\mathrm{v}}(t)$, and $\ddot{\theta}_{\mathrm{v}}(t)$

We can perform the calculation of the propulsive efficiency of the system relative to a certain time interval $\Delta t$ of working by considering the average efficiency $\eta_{m p}$. Consequently, it is necessary to evaluate the average power $P_{\text {mav }}$ consumed by the engine of the system and the average power $P_{m d}$ that the same system absorbs to move forward along a predetermined direction. In order to evaluate $P_{\text {mav }}$ we have to compute the torque $M_{\mathrm{m}}(t)$ that the driving motor applies to the cranks $r$ where the counter rotating masses are fixed. With reference to the steady state working that starts and finishes at $t=10 \mathrm{~s}$ and $t=30 \mathrm{~s}$ respectively, this torque can be computed by Eq. (47) only when it is quite sure that a steady state is established, for example, from $t=20.0 \mathrm{~s}$ to $t=20.1 \mathrm{~s} \mathrm{(} \Delta t=0.1$ s). Figs. 15 and 16 show the functions $M_{\mathrm{m}}(t)$ on the above-mentioned range, referring i) to a steady state and ii) to the systems equipped with one and two pairs of counter rotating masses, respectively. The graph of torque $M_{\mathrm{m}}(t)$ illustrated in Fig. 15 enables one to obtain the angular motion laws $\theta(t)=\theta_{\mathrm{m}}(t)=\theta_{\mathrm{v}}(t), \quad \dot{\theta}(t)=\dot{\theta}_{\mathrm{m}}(t)=\dot{\theta}_{\mathrm{v}}(t)$, and $\ddot{\theta}(t)=\ddot{\theta}_{\mathrm{m}}(t)=\ddot{\theta}_{\mathrm{v}}(t)$. Similarly, Fig. 16 illustrates the torque $M_{\mathrm{m}}(t)$ that has to be applied to the driving elliptic gear to get the graphs of

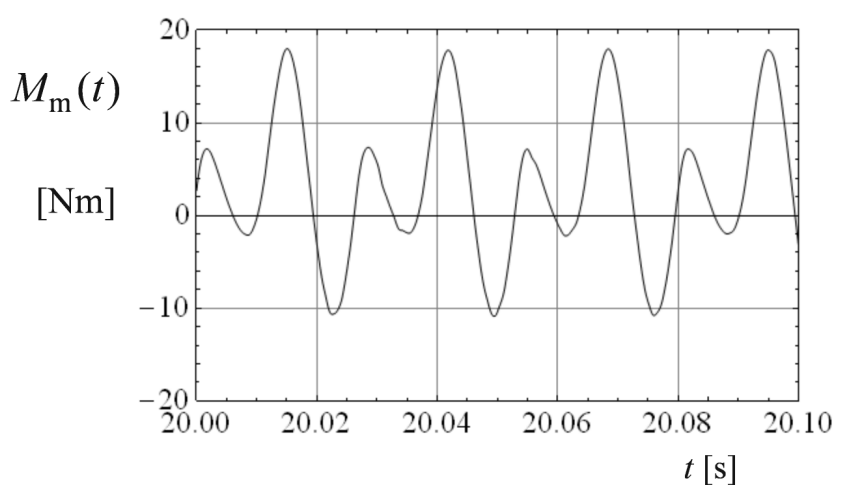

Fig. 15 Torque $M_{\mathrm{m}}(t)$ versus $20.0 \leq t \leq 20.1 \mathrm{~s}$ applied to the system equipped with one pair of counter rotating masses. 


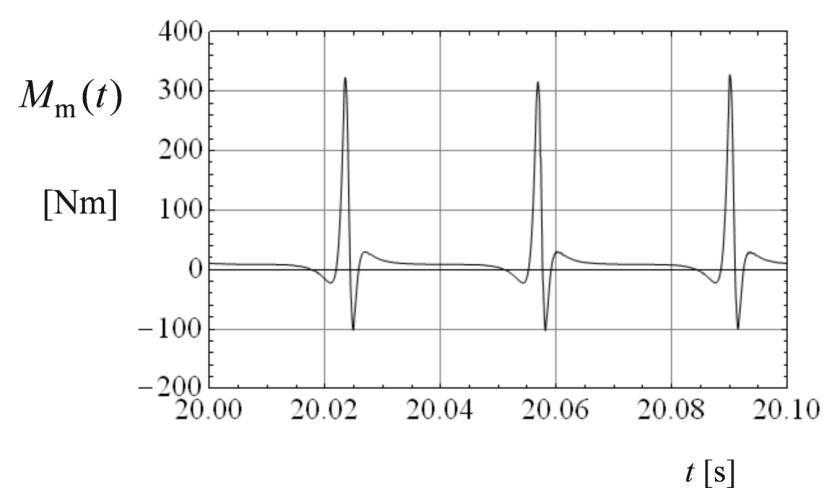

Fig. 16 Torque $M_{\mathrm{m}}(t)$ versus $20.0 \leq t \leq 20.1 \mathrm{~s}$ applied to the driving elliptical gear of the system equipped with two pairs of counter rotating masses.

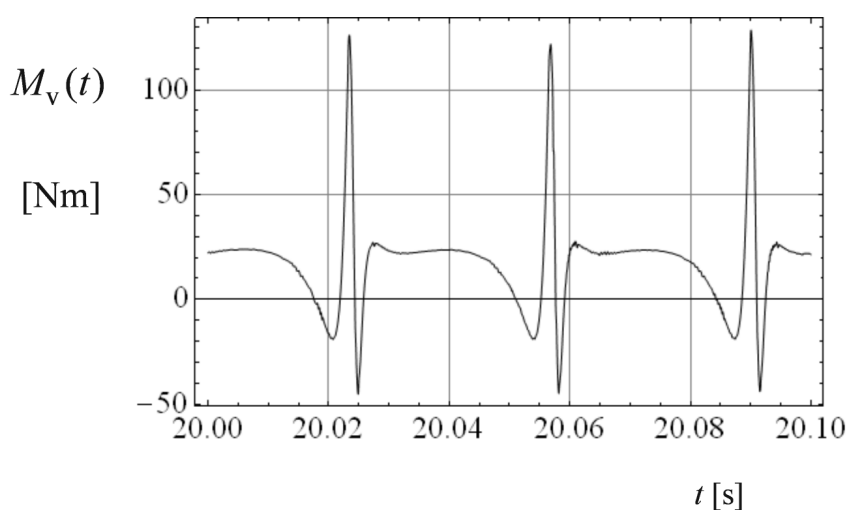

Fig. 17 Torque $M_{\mathrm{v}}(t)$ versus $20.0 \leq t \leq 20.1 \mathrm{~s}$ applied to the driven elliptical gear.

$\theta(t)=\theta_{\mathrm{v}}(t), \dot{\theta}(t)=\dot{\theta}_{\mathrm{v}}(t)$, and $\ddot{\theta}(t)=\ddot{\theta}_{\mathrm{v}}(t)$ carried out by i) integrating Eq. (30) and ii) using the functions $\theta_{\mathrm{m}}(t), \dot{\theta}_{\mathrm{m}}(t)$, and $\ddot{\theta}_{\mathrm{m}}(t)$. The torque $M_{\mathrm{v}}(t)$ applied to the driven elliptic gear can be computed by the following relationship

$$
M_{\mathrm{v}}(t) \dot{\theta}_{\mathrm{v}}(t)=M_{\mathrm{m}}(t) \dot{\theta}_{\mathrm{m}}
$$

from which

$$
M_{\mathrm{v}}(t)=M_{\mathrm{m}}(t) \frac{\dot{\theta}_{\mathrm{m}}}{\dot{\theta}_{\mathrm{v}}(t)} \quad .
$$

By assuming a constant value of $\dot{\theta}_{\mathrm{m}}$ equal to $235.619 \mathrm{rad} / \mathrm{s}$ (see Table 1) and the function $\dot{\theta}_{\mathrm{v}}(t)$ illustrated in Fig. 9(a), the graph of $M_{\mathrm{v}}(t)$ shown in Fig. 17 is obtained. The knowledge of these torques applied to the rotating parts is also useful to be able to compute the mechanical stress in the teeth of the gears meshed that have to transmit the motion.

8.2 Computation of the hydrodynamics drag force $F_{i d}(t)$

The force $F_{i d}(t)$ depends on the velocity $\dot{y}$ of the same hull, and $\dot{y}$, in its turn, depends on the instant $t$ considered. In relation to the time range from $t=20.0 \mathrm{~s}$ to $t=20.1 \mathrm{~s}$, basing on the function $F_{i d}(\dot{y})$ reported in [10], we evaluate the functions $F_{i d}(t)$ associated with the two devices studied. 


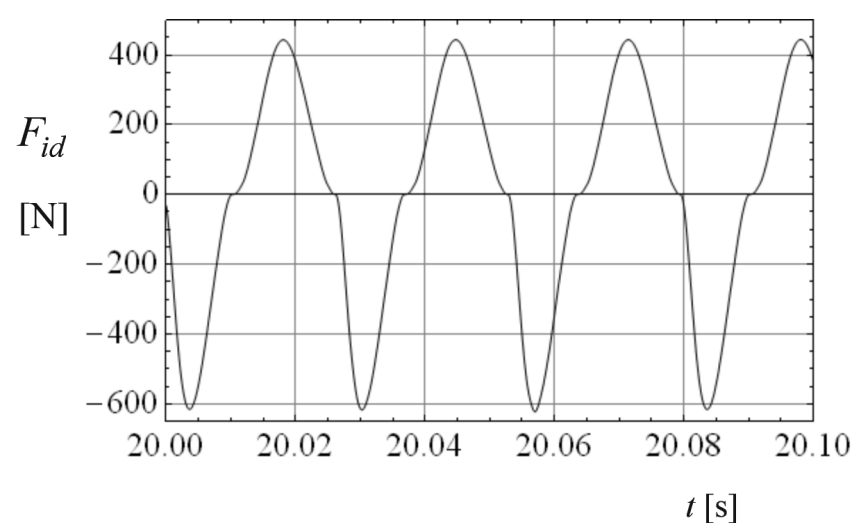

Fig. 18 Hydrodynamic drag force $F_{i d}(t)$ versus $20.0 \leq t \leq 20.1 \mathrm{~s}$ applied to the system equipped with one pair of counter rotating masses.

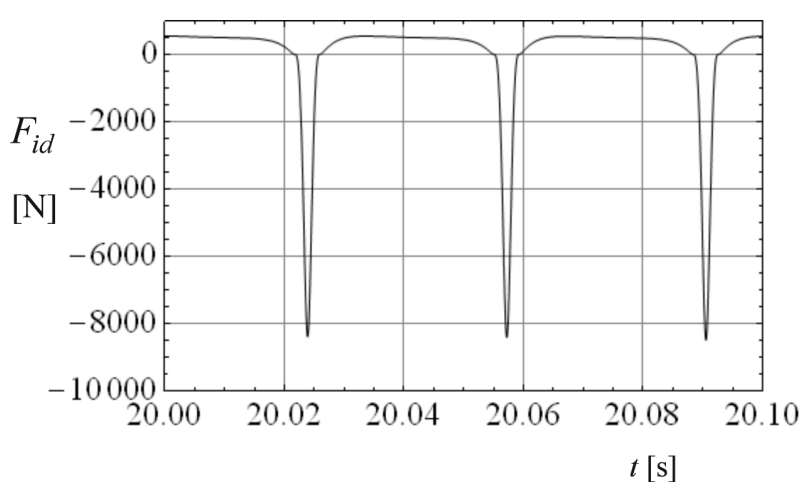

Fig. 19 Hydrodynamic drag force $F_{i d}(t)$ versus $20.0 \leq t \leq 20.1 \mathrm{~s}$ applied to the system equipped with two pairs of counter rotating masses and elliptical gears.

Figs. 18 and 19 illustrate these functions $F_{i d}(t)$ versus time. We observe that in relation to the propulsion system equipped with elliptic gears, impulsive forces $F_{i d}$ are defined. The values of these forces are very high, have minus sign, and are higher by more than ten times in respect of the lowest negative values that we notice referring to the case of propulsion obtained by a single pair of counter rotating masses.

\subsection{Physical explanation of the peaks of the torques and hydrodynamic drag force}

The peaks of the torques $M_{\mathrm{m}}(t)$ and $M_{\mathrm{v}}(t)$ are temporally coincident with those of the hydrodynamic drag force $F_{i d}(t)$ (see Fig. 19). From a physical point of view, a justification of this fact can be as follows. When the torque applied to the driving gear $M_{\mathrm{m}}(t)$ assumes maximum positive values it means that the energy supplied to the system at that instant $t$ is maximum $\left(\dot{\theta}_{\mathrm{m}}\right.$ is constant). Furthermore, observing the graph of $\dot{\theta}_{\mathrm{v}}(t)$ drawn according to kinematic considerations it is noted that the maximum values of $M_{\mathrm{v}}(t)$ occur at the same instants where $M_{\mathrm{m}}(t)$ is maximum. At the instant of the generic peak of $M_{\mathrm{m}}(t)$ and $M_{\mathrm{v}}(t)$ the energy supplied to the system causes a small amplitude backward displacement of the vessel. Nevertheless this displacement is very fast. The stern of the vessel is configured in a suitable manner and the displacement takes place with much higher speed than in the case of the device equipped with a single pair of counter rotating masses. Consequently, the undertow of the compressed water out of the stern cavity due to the backward 
Table 2. Propulsive efficiency $\eta_{m p}$, numerical values of the powers $P_{m a v}, P_{m d}$, and performances of the two propulsion systems studied.

\begin{tabular}{|c|c|c|c|c|c|c|c|c|c|c|c|}
\hline \multicolumn{6}{|c|}{$\begin{array}{l}\text { Propulsion by a single pair of } \\
\text { counter rotating masses } \\
\text { (see Figs. 1(a) and 2(a)) }\end{array}$} & \multicolumn{6}{|c|}{$\begin{array}{l}\text { Propulsion by two pair of } \\
\text { counter rotating masses driven by elliptical gears } \\
\text { (see Figs. 1(b), 2(b), and } 4)\end{array}$} \\
\hline \multirow[t]{2}{*}{$\begin{array}{l}P_{\operatorname{mav}} \\
(\mathrm{W})\end{array}$} & \multirow[t]{2}{*}{$\begin{array}{l}P_{m d} \\
(\mathrm{~W})\end{array}$} & \multirow[t]{2}{*}{$\eta_{m p}$} & \multirow[t]{2}{*}{$\begin{array}{l}\text { displacement } \\
\text { in } 40 \mathrm{~s} \\
\quad(\mathrm{~m})\end{array}$} & \multicolumn{2}{|c|}{$\begin{array}{c}\text { Mean } \\
\text { velocity } \\
\text { during the } \\
\text { steady state } \\
\text { from } \\
t=10 \mathrm{~s} \\
\text { to } \\
t=30 \mathrm{~s}\end{array}$} & \multirow[t]{2}{*}{$\begin{array}{l}P_{\operatorname{mav}} \\
(\mathrm{W})\end{array}$} & \multirow[t]{2}{*}{$\begin{array}{l}P_{m d} \\
(\mathrm{~W})\end{array}$} & \multirow[t]{2}{*}{$\eta_{m p}$} & \multirow[t]{2}{*}{$\begin{array}{l}\text { displacement } \\
\text { in } 40 \mathrm{~s} \\
\quad(\mathrm{~m})\end{array}$} & \multicolumn{2}{|c|}{$\begin{array}{c}\text { Mean } \\
\text { velocity } \\
\text { during the } \\
\text { steady state } \\
\text { from } \\
t=10 \mathrm{~s} \\
\text { to } \\
t=30 \mathrm{~s}\end{array}$} \\
\hline & & & & $(\mathrm{m} / \mathrm{s})$ & $(\mathrm{Km} / \mathrm{h})$ & & & & & $(\mathrm{m} / \mathrm{s})$ & $(\mathrm{Km} / \mathrm{h})$ \\
\hline 786.19 & 440.22 & 0.56 & 33.94 & 1.03 & 3.73 & 3743.83 & 1604.76 & 0.43 & 98.25 & 3.01 & 10.82 \\
\hline
\end{tabular}

motion cannot occur efficiently. Thus, the "ejection" of the water surrounded by the stern does not occur with the same rapidity with which it takes place in the case of the lower backward displacement caused by only a single pair of counter rotating masses. In practice, by using the elliptical wheels and two pairs of counter rotating masses, the speed of the vessel backward displacement is so high that the water in the cavity of the stern has a very short time to be pushed out from the "spoon shape" considered in [10]. In this situation, in correspondence to the stern a real constraint is almost defined (the water is practically incompressible). The constraint generates a particularly intense impulsive reaction force. This reaction is $F_{i d}$ and its sign is negative (see the graph in Fig. 19), i.e. $F_{i d}$ pushes the vessel forward. As a matter of fact, it is noted that the second member of the corresponding motion equation of the system (14), is $-F_{i d}$ : then, if $F_{i d}$ is negative (see the peaks in the graph shown in Fig. 19) it means that, at the instant of the peak, $-F_{i d}$ has the maximum value and pushes the vessel forward.

\subsection{Computation of the propulsive efficiency}

The propulsive efficiency $\eta_{m p}$ of the device equipped with counter rotating masses can be conveniently evaluated by Eq. (35). $\eta_{m p}$ has been computed with reference to the time range from $t$ $=20.0 \mathrm{~s}$ to $t=20.1 \mathrm{~s}(\Delta t=0.1 \mathrm{~s})$. Therefore the average power $P_{\text {mav }}$ consumed by the engine of the system and the average power $P_{m d}$ that the same system absorbs to move forward along a predetermined direction on the time interval $\Delta t$ were calculated. This numerical computation was performed referring to a steady state working. The results obtained relative to the two propulsion systems comparated, i.e. those equipped with a single and two pairs of counter rotating masses (the second one is also driven by ellipical gears), are shown in Table 2 . We observe that in both cases the propulsive efficiency $\eta_{m p}$ is rather high: 0.56 and 0.43 in the first and in second case, respectively. We can justify the fact that the second efficiency is lower than the first one because the hull equipped with elliptic gears is characterized by higher mean forward velocity: the higher the velocity of the vessel is, greater is the dissipation of energy due to hydrodynamic drag force which does not linearly increase versus $\dot{y}$. 


\section{Conclusions}

In this paper equations to study the vibratory behavior in hulls devoid of propeller with the aim of obtaining an efficient forward motion are obtained. The propulsion system provides the installation on board of vessels of a device able to generate centrifugal forces. The motion is achieved by virtue of a suitable configuration of the stern of the hull. The system, during the instants in which it is coming back, must be subject to a hydrodynamic drag force higher than that rises during the forward motion. The centrifugal force generated by the counter rotating masses assembled on board makes the entire system oscillate back and forward. The difference in the hydrodynamic drag force generated by the motion along the two opposite directions of translation determines the prevalence of the forward motion with respect to the backward one. In relation to possible practical applications of the propulsion system, definitely an important parameter to consider is the propulsive efficiency of the system. The study illustrates how this efficiency can be calculated. Successively, the results of numerical simulations based on the analysis developed are presented. The results obtained can be considered rather good because, in relation to the size of the system studied, the propulsive efficiencies obtained are definitely higher than those obtainable by conventional propellers of small dimensions. Moreover, the numerical simulations have shown that it is possible to considerably increase the forward speed of the vessel if i) the rotation of the counter rotating masses does not occur with constant angular velocity during each turn and ii) the single pair of masses is replaced by two pairs of masses. In particular the case of a device with two pairs of counter rotating masses driven by elliptical gears has been studied. The utilisation of elliptical gears causes impulses of angular velocity of the counter rotating masses during the single revolution made by the same ones. An important parameter that has been identified to maximize the distance that the vessel covers is represented by the phasing angle $\Delta$. This angle characterizes the relative angular position of the two pairs of counter rotating masses. The numerical results obtained relative to the device equipped with two pairs of counter rotating masses driven by elliptical gears and $\Delta=$ 357.8 degree $(6.245 \mathrm{rad})$, suggest that a particular condition of excitation could determine a kind of tuning in the nonlinear steady working of the water-hull system. In this situation a predominance of the amplitudes of oscillation of the "hull-part" of the system water-hull along the forward direction compared to the backward one happens. From an engineering application point of view we observe that before we manufacture a real prototype of the system, it is wise to evaluate the propulsion efficiency in relation to vessels whose size is greater than that considered in this case study. We are developing a simulation with reference to a hull $14 \mathrm{~m}$ long, already manufactured and equipped with a conventional propeller propulsion system. With reference to this hull a virtual model with a suitably modified stern was made. New curves of the hydrodynamic drag force $F_{i d}$ versus the velocity $\dot{y}$ similar to those reported in [10] have already been obtained and a study of a device with multiple counter rotating masses suited to meet the needs of engineering is in progress. Other aspects that should be considered from an engineering point of view are those relating to the vibrations of the whole structure of the hull. Since the excitation frequencies are relatively high compared to the natural vibration frequencies of a real hull, one can reasonably expect that the vibration problems related to the structural safety and comfort will be overcome.

\section{References}

1. Chernousko, F.L., 2002. The optimum rectilinear motion of a two-mass system, Prikl. Mat. Mekh., 66 (1), pp. 3-9.

2. Chernousko, F.L., 2005. The motion of a body containing a mobile internal mass, Doklady Akademii Nauk SSSR, 405 (1), pp. 56-60.

3. Chernousko, F.L., 2006. Analysis and optimization of the motion of a body controlled by means of a movable internal mass. Prikl. Mat. Mekh., 70, (6), pp. 915-41. 
4. Chernousko, F.L., 2008. The optimal periodic motions of a two-mass system in a resistant medium. Journal of Applied Mathematics and Mechanics,72, pp. 116-25.

5. Elleri A., 2010. Study and development of a non-conventional propulsion system for boats and land vehicles (in Italian), Master Thesis, Supervisor: R. Muscia, University of Trieste, Italy.

6. Fang, H., and $\mathrm{Xu}, \mathrm{J}, 2012$. Dynamics of a three-module vibration-driven system with nonsymmetric Coulomb's dry fiction. Multibody System Dynamics, 27, pp. 455-485.

7. Fang, H., and Xu, J., 2011. Dynamics of a mobile system with an internal acceleration-controlled mass in a resistive medium. Journal of Sound and Vibration, 330, pp. 4002-4018.

8. Li, H., Furuta, K., and Chernousko F.L., 2005. A pendulum-driven cart via internal force and static friction. Proceedings of the International Conference "Physics and Control", Saint Petersburg, Russia, pp. 7-15.

9. Li, H., Furuta, K., and Chernousko F.L., 2006. Motion generation of the Capsubot using internal force and static friction. Proceedings of the 45th IEEE Conference on Decision and Control. San Diego, CA, USA, pp. 6575-80.

10. Muscia, R. and Sciuto, G., 2010. Analytic study of a new conceptual propulsion device for ships, International Journal of Naval Architecture and Ocean Engineering, 2 (2), pp. 75-86.

11. Shaw, S.W., 1986. On the dynamic response of a system with dry friction. Journal of Sound and Vibration, 108 (2), pp. 305-325.

12. Yang, L., Wiercigroch, M., Pavlovskaia, E., and Yu, H., 2013. Modelling of a vibro-impact capsule system, International Journal of Mechanical Sciences, 66, pp. 2-11.

13. Chen, H.C. and Lee, S.K., 2003. Time-domain simulation of propeller-ship interactions under turning conditions. Proceedings of the $16^{\text {th }}$ ASCM Engineering Mechanics Conference, USA, University of Washington, Seattle, pp.16-18.

14. Couser, P., Wellicome, J.F., and Molland, A.F., 1998. An Improved Method for the Theoretical Prediction of the Wave Resistance of Transom Stern Hulls using a Slender Body Approach. International Shipbuilding Progress, 45(444), pp 331-349.

15. Dai, C.M.H., Gorski, J.J., and Haussling, H.J., 1991. Computation of an integrated ducted propulsor/stern performance in axisymmetric flow. Proceedings of the SNAME Propeller/Shafting'91 Symposium, USA, Virginia Beach, VA, pp. 14.1-14.12.

16. Dhinesh, G., Murali, K., and Subramanian, V. A., 2010. Estimation of hull-propeller interaction of a self propelling model hull using a RANSE solver. Ships and Offshore Structures, 5 (2), pp. $125-139$.

17. Lee, S.K., Liao, M., and Wang, S., 2006. Propeller-Induced Hull Vibration - Analytical Methods. Proceedings of the $2^{\text {nd }}$ International Ship Noise and Vibration Conference, UK, London, pp. 127-139.

18. Rijpkema, D., Starke, B., and Bosschers, J., 2013. Numerical simulation of propeller-hull interaction and determination of the effective wake field using a hybrid RANS-BEM axpproach. Proceedings of the $3^{\text {rd }}$ International Symposium on Marine Propulsors, Australia, Launceston, Tasmania.

19. Stern, F., Kim, H.T., Patel, C., and Chen, H.C. 1988b. A viscous-flow approach to the computation of propeller-hull interaction. Journal of Ship Research, 32 (4), pp. 246-262.

20. Stern, F., Toda, Y., and Kim, H.T., 1991. Computation of Viscous low Around PropellerBody Configurations: Iowa Axisymmetric Body. Journal of Ship Research, 35 (2), pp. 151161.

21. Zhang, D.H., Broberg, L., Larsson, L., and Dyne, G., 1992. A method for computing stern flows with an operating propeller. Royal Institution Naval Architects, 134.

22. Mathematica, Wolfram Research, Champaign, USA, IL - WorldwideHeadquarters. 